\title{
Carbon nanotubes prepared by anodic aluminum oxide template method
}

\author{
HOU PengXiang, LIU Chang*, SHI Chao \& CHENG HuiMing \\ Shenyang National Laboratory for Materials Science, Institute of Metal Research, Chinese Academy of Sciences, Shenyang 110016, China
}

Received August 9, 2011; accepted September 15, 2011; published online December 1, 2011

\begin{abstract}
One of the most unique structural characteristics of carbon nanotubes (CNTs) differentiating from other carbon materials is their hollow nanochannles, which can be utilized for encapsulating and loading foreign matters. The anodic aluminum oxide (AAO) template technique enables the diameter, length, and cap structure control of the replicated CNTs, and thus shows advantages in pore structure control over the traditional CNT growth approaches. This review details the synthesis of CNTs with tunable diameter, length, wall thickness, and crystalline by using the AAO template method. The doping of heteroatoms and filling of foreign matters into AAO-CNTs are also addressed. Moreover, the main challenges and developing trends of the AAO template method are discussed.
\end{abstract}

carbon nanotubes (CNTs), anodic aluminum oxide (AAO) template method, controllability

Citation: Hou P X, Liu C, Shi C, et al. Carbon nanotubes prepared by anodic aluminum oxide template method. Chin Sci Bull, 2012, 57: 187-204, doi: $10.1007 / \mathrm{s} 11434-011-4892-2$

Carbon nanotubes (CNTs) with unique structure and properties such as well-defined hollow interiors, inert surface properties, and resistance to acid and base environment have been proven to be excellent candidates as support in catalytic reactions [1-7] and energy storage and conversion medium [8-11]. Structural parameters of CNTs such as inner diameter, wall thickness, length, crystallinity, and electronic structure have a great influence on the performance of the supported particles or loaded matters [12-15]. Especially, the size of the CNT hollow core and electron density plays an important role in determining the property of the filled matters owing to the confinement effect and the difference in electron density between the exterior and interior CNT surface due to their different graphene curvature $[14,16]$. Some new phenomena were reported for the materials confined in CNT nanochannels, which have never been observed for their bulk counterparts [17-19]. The physics and chemistry of materials confined within a one-dimensional nanochannel are receiving increasing research interest. To systematically investigate the function, and working

*Corresponding author (email: cliu@imr.ac.cn) mechanism of the one-dimensional space confinement effect, it is essential to synthesize CNTs with high purity and finely controlled structures, such as desired diameter, length, wall thickness, and electronic structure. However, it is difficult to synthesize pore-texture controllable and impurityfree CNTs using the conventional synthesis methods, such as chemical vapor deposition (CVD) [20], laser ablation [21], and arc discharge method [22], due to the lack of understanding on the growth mechanism and corresponding controlled growth techniques. In addition, cutting and opening the cap of CNTs is usually necessary before filling process, which is complex and may introduce defects and contaminations into CNTs. Furthermore, it is very difficult to ensure a uniform length and opening rate of CNTs by using the above approaches. Another knotty problem is that some particles will unavoidably deposit on the outer surface rather than fill into the inner cavity of CNTs, which makes it difficult in investigating the confinement effect of CNTs due to the complex locations and states of the foreign matters. Although considerable efforts have been made [23-28], these above problems have not been well solved yet. On the other hand, template carbonization technique provides an 
alternative way for the precisely controlled synthesis of CNTs with desired diameter, opened cap, high purity, and selective filling of foreign matters inside the hollow cores.

The template carbonization method consists of carbonization of an organic gas or polymer in nanospace of an inorganic template and liberation of the deposited carbon from the template [29]. Anodic aluminum oxide (AAO) film is synthesized by the anodization of aluminum metal in acidic media. The AAO film has uniform and straight nanosize channels with a tailored length and diameter [30,31]. Using these uniform and straight nanochannels of AAO film as a template, Kyotani et al. [32,33] first prepared CNTs free of carbonaceous and metal impurity by the deposition of pyrolytic carbon onto the channels of the AAO film. The length, diameter and density of the as-synthesized CNTs can be uniformly tailored because of the controllability of the pore texture of AAO template [34-36]. Furthermore, the wall thickness and crystallinity of the CNTs can be controlled by adjusting CVD conditions, such as the composition and concentration of carbon source and the temperature and period of CVD process $[33,37,38]$. Another unique advantage of the template method is that it allows complete filling of foreign materials inside the hollow core of CNTs. The size, amount, and distribution of the filled matter can be controlled by tuning the filling parameters. In addition, the AAO template synthesis method makes it possible to selectively dope or chemically functionalize the inner or outer surface of CNTs. Owing to these unique characteristics, the CNTs prepared by AAO template method may find applications in biomedical or drug delivery [39], small molecule delivery systems [40], high density magnetic recording [41], anode materials [42], etc.

Although the CNTs synthesized by the AAO template method have many unique characteristics, it shows also some limitations such as high cost, wavy carbon layer structure, relative large diameter, etc.

In this article, we are to give a comprehensive overview on the synthesis of CNTs by the AAO template method. And the CNTs synthesized by AAO template method were denoted as "AAO-CNTs" hereafter. We focus on describing the controllability of the pore texture, heteroatom doping, and filling of foreign matters into the inner hollow core of CNTs by the AAO template method. The challenges and developing trends of the AAO template method are also discussed. The objective of this article is to show the effectiveness of preparing CNTs with specific structures by using the AAO template method, to present the efficient approach of filling foreign matters inside CNTs, and to stimulate innovative thoughts on the preparation and application of AAO-CNTs.

\section{Preparation of AAO-CNTs}

Template carbonization method represents a conceptually straightforward approach. In this method, the template simply serves as a structural framework within which carbon material can be generated by carbonization from precursors in situ and shaped into a nanostructure with its morphology dictated by the size and shape of the template scaffold [43]. For the synthesis of CNTs, porous AAO film is an ideal choice for templating, because of their parallel and straight channels, highly uniform distribution of cylindrical pores arranged in a hexagonal array, and tunable pore length, diameter and interpore distance. Furthermore, AAO films are thermally and mechanically stable, which makes their structure stable even at high carbonization temperature of $900^{\circ} \mathrm{C}$ under inert gas atmosphere [44]. Therefore, controlled synthesis of CNTs using AAO as template has been widely investigated. The controlled preparation of AAO-CNTs will be elucidate in detail in the following section.

\subsection{AAO template}

The formation of an alumina film on aluminum surface is a well know process, which happens in air spontaneously. Highly ordered AAO films are obtained by anodically oxidizing aluminum plate in a strong acid electrolyte (such as sulfuric, oxalic, phosphoric and chromic acid solutions), in which a self-organized process occurs [44,45]. The selforganized anodic porous alumina existed as a closely packed array of hexagonally arranged cells containing pores in each cell center (Figure 1). The highly ordered nanochannel structure is usually characterized by given parameters of uniform pore diameter, length and interpore distance. The pore diameter, which can be controlled by altering the anodizing conditions, are in the range of a few nanometers to hundreds of nanometers. Generally, the pore diameter is linearly proportional to the anodizing potential with a proportionality constant of approximately $1.29 \mathrm{~nm} \mathrm{~V}^{-1}$ [46]. It is also related to the current density, electrolyte temperature, and acid concentration [45-48]. The depth (length) of the parallel AAO channels can be controlled in the range of 0.1-300 $\mu \mathrm{m}$, which is mainly determined by the anodizing period. Due to the hexagonal symmetry of the cells, anodic porous alumina

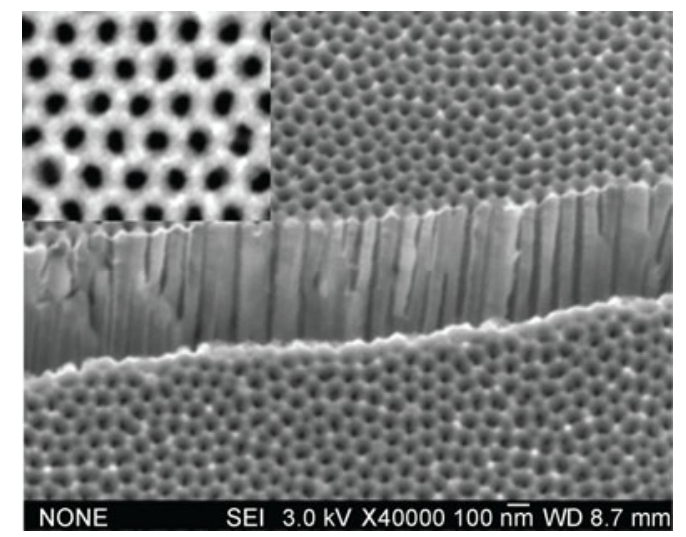

Figure 1 SEM image of an AAO film, inset is the enlarged SEM image. 
is a nanostructure with high packing density of $10^{8}-10^{11}$ pores per $\mathrm{cm}^{2}$ [49-52]. Therefore, the CNTs replicated from the AAO template are also highly ordered arrays with controllable diameters and lengths.

Another important morphological feature of AAO film is its wall thickness, which determines the intertube distance of templated CNTs. It is generally accepted that the wall thickness is linearly proportional to the forming potential of the steady-state growth of anodic porous alumina, while it is also related to the electrolyte temperature, acid concentration and acid type used. And the wall thickness of AAO film is popularly in the range of $10-350 \mathrm{~nm}$, in consequence the intertube distance of AAO-CNTs would be larger than that of the CNT arrays synthesized by conventional methods [53,54].

In general, the formed AAO film remains attached to the Al substrate, with one end of the nanochannels open and the other closed by an oxide barrier layer (Figure 2(a)). The barrier layer can be further detached from the Al base to obtain free standing AAO film as shown in Figure 2(b). There are mainly three methods for separating the AAO film from remaining Al substrate. One way is to use an electrochemical detachment method, where a reverse voltage is applied [33,55]. The second is to perform electrochemical etching in $20 \% \mathrm{HCl}$ with an operating potential of 1-5 V [56]. The third and also the most frequently used method is to employ a wet chemical process, in which the unoxidized aluminum substrate is dissolved by immersing an anodized sample into a saturated solution of $\mathrm{HgCl}_{2}, \mathrm{CuCl}_{2}$, $\mathrm{CuSO}_{4}, \mathrm{CuCl}_{2} / \mathrm{HCl}$, etc. [57-60]. The barrier layer can also be removed to obtain two side open AAO film as shown in Figure 2(c). Chemical etching is often used to dissolve the barrier layer, for example by immersing into a $\mathrm{H}_{3} \mathrm{PO}_{4}$ solution, and the opening time depends directly on the barrier layer thickness [61,62]. However, pore-widening often occurs due to the difficulty in precisely control the degree of barrier layer etching. $\mathrm{Xu}$ et al. [63] demonstrated that $\mathrm{Ar}^{+}$ ion milling at grazing incidence could open the nanopores from the "closed side" in a controlled manner. Recently, a new technique was developed to simultaneously remove $\mathrm{Al}$ substrate and barrier layer using a short time electrical oxidation with voltage pulse of about $5 \mathrm{~V}$ higher than that for anodic oxidation in a solution of $\mathrm{HClO}_{4}$ and $\left(\mathrm{CH}_{3} \mathrm{CO}\right)_{2}$
[64,65]. After this process, free standing and through-hole AAO can be obtained immediately, and there is no damage to the channels of the AAO film.

Therefore, the length, diameter, and cap structure of the channels of AAO template can be easily tuned, which facilitates the structure controlled preparation of CNTs when using AAO as a template.

\subsection{Synthesis of CNTs by the AAO template method}

The process of preparing CNTs using AAO as template is illustrated in Figure 3. A carbon layer is first deposited onto the AAO film, then the template is removed and freestanding CNT arrays are obtained. There are two approaches to deposit carbon onto the nanochannel surface of AAO template. One is to carbonize organic polymer (such as furfural alcohol and acrylonitrile) that was filled into the nanochannels of AAO using impregnation method [33,66,67]. The second is to deposit pyrolytic carbon from gaseous hydrocarbons (acetylene, propylene, acetonitrile, etc), which is much easier and widely used now [32,68-73]. Typically, a gaseous carbon source is decomposed within a hot-wall tubular reactor in which the AAO film is preset. The thermal decomposition of gaseous carbon source results in the uniform deposition of carbon layers onto the surface of the nanochannels in the AAO film. After a suitable period of time, the reactor is cooled down to room temperature and the AAO template is removed by immersing in $\mathrm{NaOH}$ or HF acid solution. Following the above preparation process, the length, outer diameter, cap structure (close or open), and intertube distance of the obtained CNTs are well replicated from the AAO nanochannel structure. The wall thickness can be tuned to some extent by controlling the CVD conditions. And the CNTs obtained are catalyst free and carbonaceous impurity free. At the same time, it is much easier to selectively fill foreign materials into the AAO-CNTs due to their open end and unique preparation procedure.

(1) Length control of AAO-CNTs. The length of AAOCNTs is determined by the nanochannel length of AAO template, i.e. the thickness of AAO films, which can be controlled by tuning the anodizing time. We thus prepared AAO-CNTs with lengths of 60,5 , and $0.2 \mu \mathrm{m}$, and their SEM images are shown in Figure 4(a)-(c), respectively. It

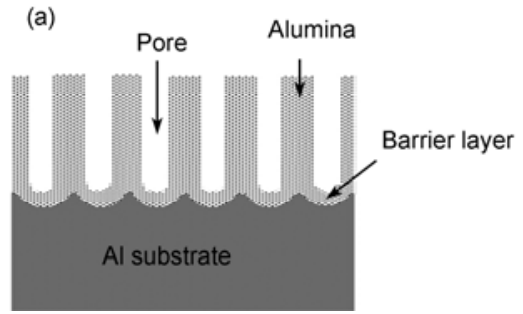

(b)

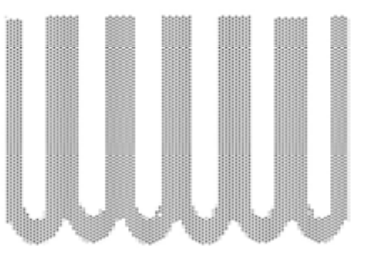

(c)

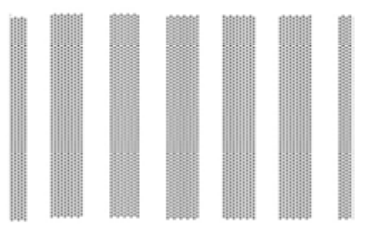

Figure 2 Schematic diagram of (a) an AAO film attached to the Al substrate; (b) a free-standing AAO film with one channel end close; (c) a free-standing AAO film with both channel ends open. 


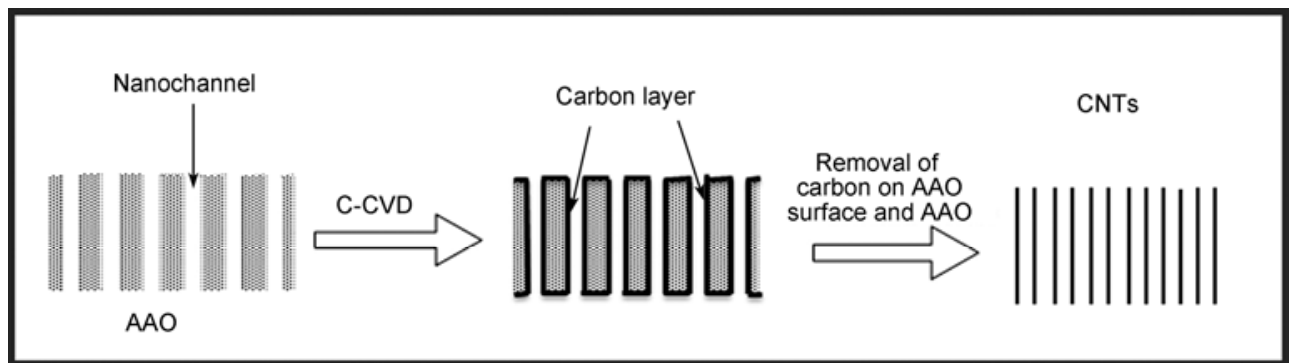

Figure 3 Schematic drawing of the formation process of AAO-CNTs.
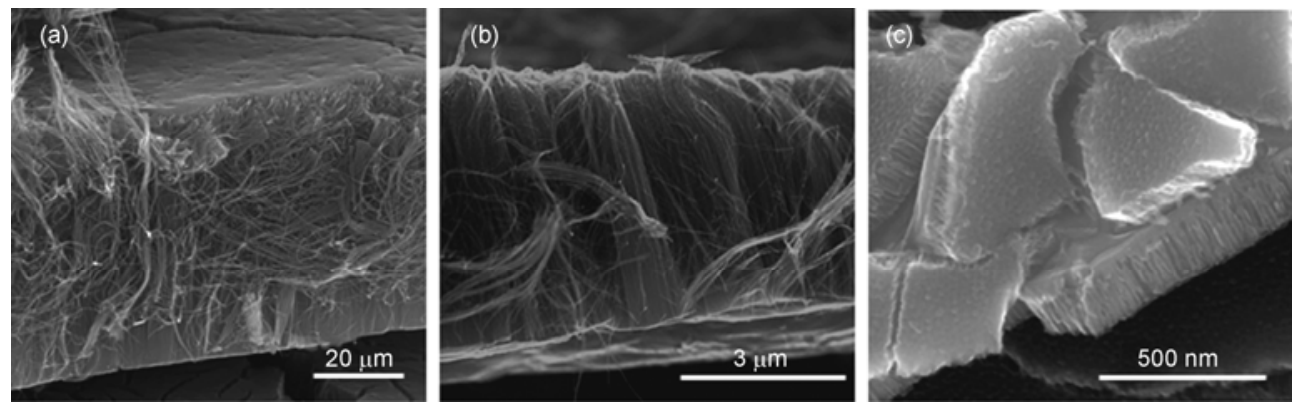

Figure 4 SEM images of the AAO-CNT arrays with lengths of (a) $60 \mu \mathrm{m}$, (b) $5 \mu \mathrm{m}$, and (c) $0.2 \mu \mathrm{m}$.

can be seen that the CNTs assemble to densely packed arrays, and the lengths of the CNTs are rather uniform. The length of AAO-CNTs can be as long as $300 \mu \mathrm{m}$. The preparation of the anodic alumina film is a result of equilibrium between the formation and dissolution of aluminum oxide $[74,75]$. In general, the dissolution rate of oxide is lower than the formation process of the oxide. This makes the pore sizes of the AAO film at two ends are not strictly same, especially when the channels are very long. Therefore, the length of AAO-CNTs reported by now is generally shorter than $100 \mu \mathrm{m}$. And the shortest AAO- CNTs reported are about $0.5 \mu \mathrm{m}$ [76]. The difficulty in getting even shorter AAO-CNTs lies in the controlled preparation of very thin and free standing AAO films. In addition, before the removal of AAO template by acid or alkali immersion, the carbon layer coated on the upper surface of the film needs to be removed by an oxygen plasma irradiation treatment. When the AAO film is very thin, the carbon coated onto the surface of nanochannels would be partially etched away during the oxygen plasma irradiation treatment, which results in un-uniformity of the obtained AAO-CNTs.

(2) Diameter control of AAO-CNTs. Both the outer diameter and inner diameter of AAO CNTs can be tuned. The outer diameter of AAO-CNTs equals to the channel diameter of the AAO template. By selecting anodizing voltage at different electrolytes during the anodizing process, AAO films with different pore sizes can be obtained. For example, $\mathrm{H}_{3} \mathrm{PO}_{4}, \mathrm{C}_{2} \mathrm{H}_{2} \mathrm{O}_{4}$, and $\mathrm{H}_{2} \mathrm{SO}_{4}$ electrolytes produce AAO films with pore sizes of $150-300 \mathrm{~nm}, 30-70 \mathrm{~nm}$, and below $20 \mathrm{~nm}$, respectively [68,77]. Now, AAO films with a uniform and adjustable channel sizes of 20-200 nm can be reproducibly synthesized [78]. As a result, AAOCNTs with outer diameters ranging from 20 to $200 \mathrm{~nm}$ can be easily prepared [46,47,79-81]. AAO film with channel size smaller than $20 \mathrm{~nm}$ can also be synthesized by selecting low voltage or in dilute sulfuric acid at low temperature $\left(\right.$ near $0^{\circ} \mathrm{C}$ ) [76,82]. SEM images of the AAO-CNTs with outer diameters of 100, 30, and $10 \mathrm{~nm}$ are shown in Figure 5.
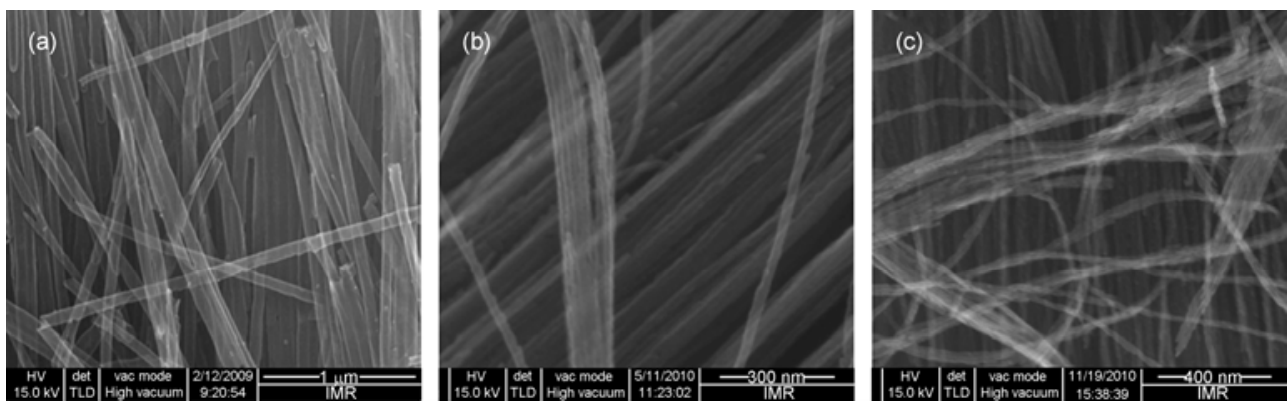

Figure 5 SEM images of AAO-CNTs with outer diameters of (a) $100 \mathrm{~nm}$, (b) $30 \mathrm{~nm}$, and (c) $10 \mathrm{~nm}$. 
It can be seen that the outer diameters of the CNT samples are rather uniform, which is an important characteristics of AAO-CNTs.

The inner diameter of AAO-CNTs can be controlled using two methods. One way is to adjust the CVD conditions. For example, by increasing the CVD time, the wall thickness of CNTs with given outer diameter can be increased, and their inner diameter will be decreased. The CNTs synthesized by this way have same outer diameter and tunable wall thickness and inner diameter (as shown in Figure 6). Since the outer diameter of AAO-CNTs can be as large as $300 \mathrm{~nm}[68,77]$, their inner diameter can be tuned in a wide range of several $\mathrm{nm}$ to nearly $300 \mathrm{~nm}$. The other approach is to select AAO films with different channel sizes as template. The CNTs thus synthesized may have varied outer and inner diameters in a wide range. However, it is still difficult to obtain AAO-CNTs with an inner diameter smaller than $4 \mathrm{~nm}$, possibly due to the difficulty in the diffusion of carbon source in very tiny channels.

A unique characteristic of the AAO-CNTs is their uniformity in both outer and inner diameters. This uniformity can be characterized by either direct TEM observation or by pore size distribution measured from cryo- $\mathrm{N}_{2}$ adsorption/ desorption technique. As shown in Figure 7(a), AAO-CNTs with uniform outer diameter, wall thickness, and inner diameter can be clearly seen from the TEM observation. And their outer and inner diameters are around 20 and $15 \mathrm{~nm}$, respectively. The pore size distribution of the AAO template and the corresponding AAO-CNTs plotted from $\mathrm{N}_{2}$

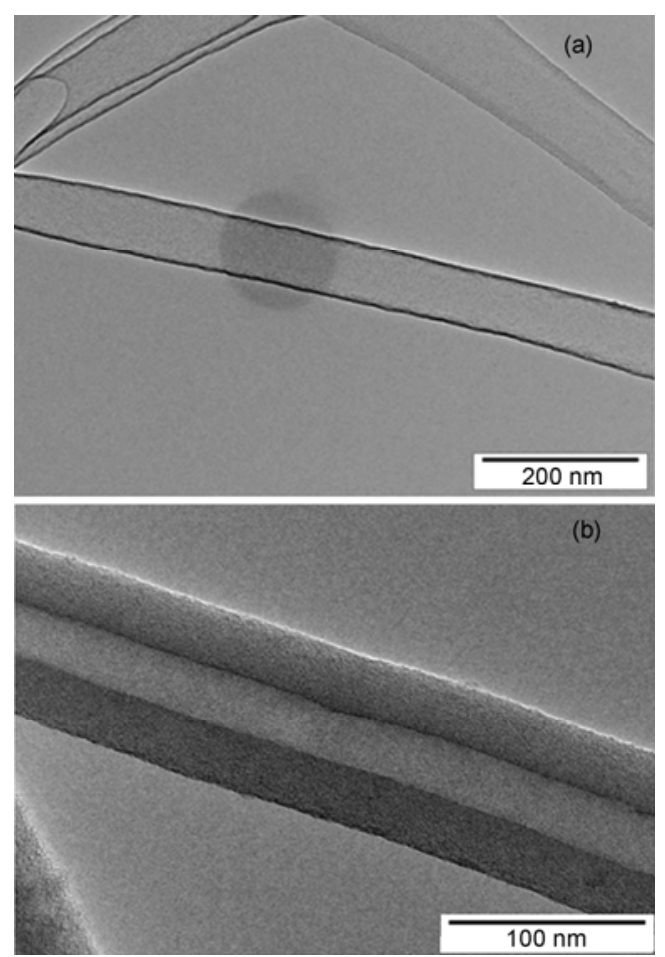

Figure 6 TEM images of AAO-CNTs with an outer diameter of $\sim 80 \mathrm{~nm}$ and different inner diameters of $\sim 70 \mathrm{~nm}$ (a) and $\sim 27 \mathrm{~nm}$ (b).
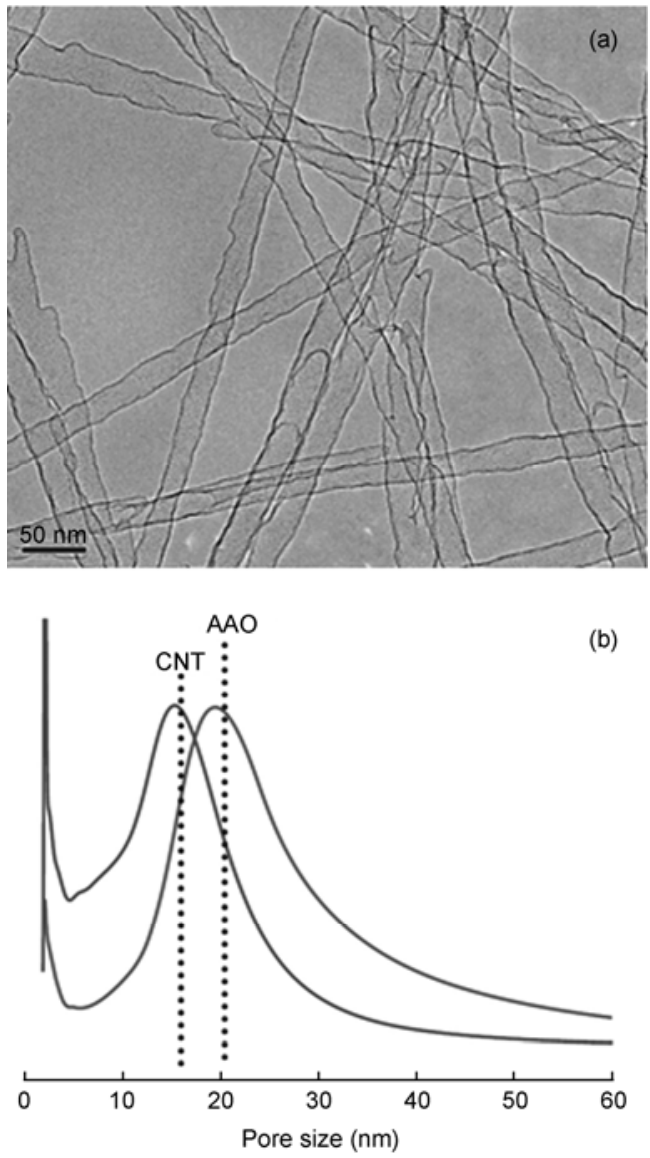

Figure 7 (a) TEM image of AAO-CNTs with outer and inner diameters of $\sim 20$ and $15 \mathrm{~nm}$, respectively; (b) pore size distributions of the AAO template and corresponding AAO-CNTs obtained from $\mathrm{N}_{2}$ adsorption isotherm by BJH theory.

adsorption isotherm based on BJH theory are shown in Figure 7(b). It can be seen that the pore size of the AAO template is narrowly distributed and centered at $\sim 20 \mathrm{~nm}$, which roughly equals to the outer diameter of the AAO-CNTs shown in Figure 7(a). The pore size, i.e. the inner diameter of the AAO-CNTs is centered at $\sim 15 \mathrm{~nm}$. Therefore, the outer and inner diameters of the AAO-CNTs obtained from microscopic TEM observation and statistical cryo-nitrogen adsorption characterization are in good agreement.

(3) Cap control of AAO-CNTs. The CNTs synthesized by conventional methods of arc discharge, laser ablation, and CVD [83-85] are usually cap-closed. Because the nanochannels of AAO films can be either one-side open or both-side open $[30,31,86]$, the cap structure of AAO-CNTs can be controlled with good uniformity by selecting different types of AAO templates [33,38].

Figure 8(a) and (b) show SEM images of a both-side open AAO template. Many both-side open channels with uniform diameters are observed on both the top (Figure 8(a)) and bottom side of the AAO film (Figure 8 (b)). And the bottom side of a one-side open AAO film is shown in Figure 8(c) for comparison. Therefore, it is easy to understand 

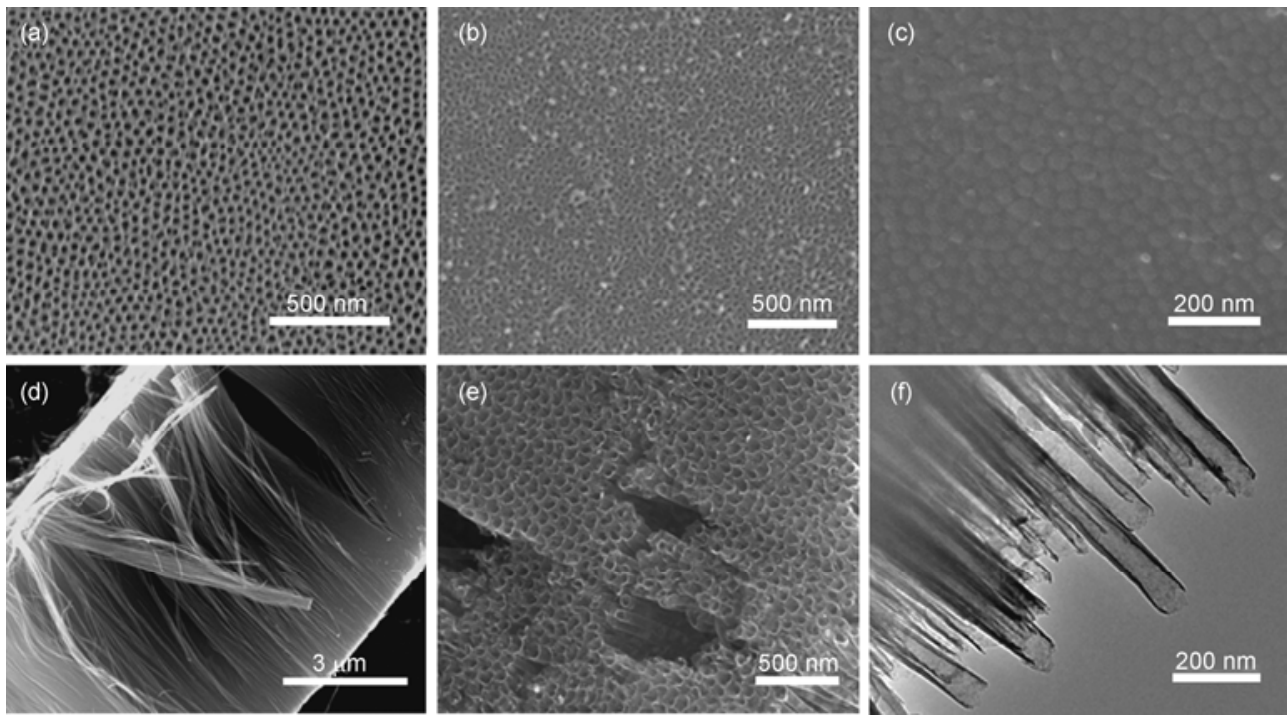

Figure 8 SEM images of (a) top-side view of a both-side open AAO film; (b) bottom-side view of a both-side open AAO film; (c) bottom-side view of a one-side open AAO film; (d) both-side open AAO-CNTs; (e) a high magnification image of (d); (f) TEM image of the two-side open AAO-CNTs.

the AAO-CNTs can be either one-side open or two-side open, which is determined by the structure of the employed AAO film template. Figure 8(d) shows SEM image of a both-side open AAO-CNT sample. It can be seen that the CNTs pack into a tidy array and their lengths are very uniform. Figure 8(e) and (f) are enlarged SEM image and TEM image of the CNT cap shown in Figure 8(d). It can be seen that the caps of the CNTs are uniformly open with similar cap size and morphology.

(4) Morphology control of AAO-CNTs. The morphology of the AAO-CNTs are not limited to be tubular-shape, Y-shape and hierarchically branched CNTs have also been reported [87-91]. Li et al. [87] first synthesized well aligned Y-junction CNTs using an AAO film with Y-branched nanochannels as template. Meng et al. [88,89] reported a method for preparing AAO template with a hierarchically branched structure (as shown in Figure 9). After the initial anodization to form the stem pores, the anodizing voltage was reduced by a factor of $1 / \sqrt{n}$, transforming a linear pore into a multiply branched pore. By sequentially decreasing the voltage by a factor of $1 / \sqrt{m}$, the authors generate the second-generation multibranched pores growing from each of the first-generation multibranched pores. The number, frequency, diameter and length of the branches were controlled through the voltage and duration of anodizing. It is predicted that the CNTs with Y shape and multiple branches yield a wealth of new architectures and could be used to design nanoscale biomaterials and electronic circuits [89].

\section{Hetero atom doping of the AAO-CNTs}

The CNTs consisting of pure carbon are non-polar, which limits their applications to some extent. The introduction of

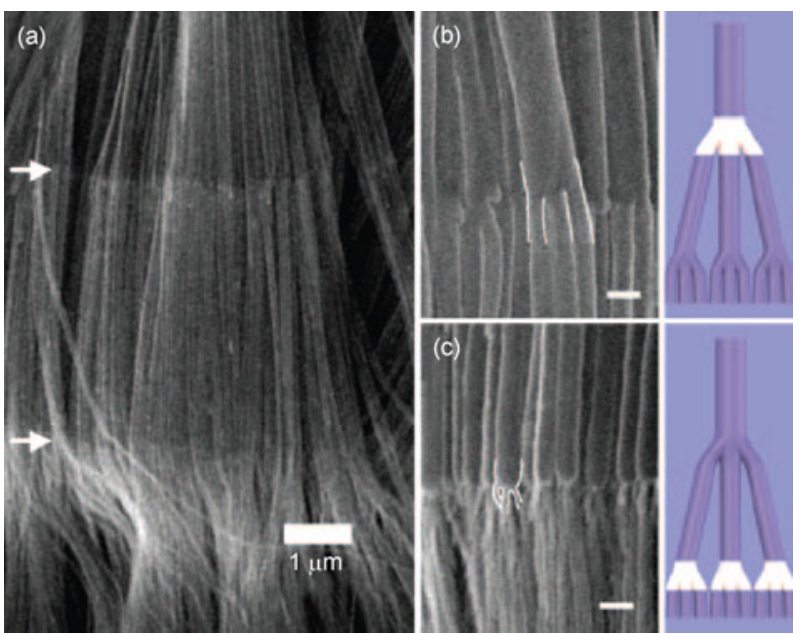

Figure 9 CNT architectures that have complex hierarchical branching. (a) SEM image of CNT arrays showing two generations of branching (at the locations of the white arrows); ((b) left and (c) left) close-up views of the $\mathbf{1}$ $\rightarrow \mathbf{3}$ junctions, ((b) right and (c) right) schematic of each of the representative individual nanotube structures. Junctions are highlighted with white line contours for clarity (scale bars: $100 \mathrm{~nm}$ ) [88].

hereroatoms such as $\mathrm{N}$ or B endows CNTs with polar nature, and their physico-chemical properties would thus be different from those of pure CNTs. At the same time, doping of heteroatoms into graphene layers constituting CNTs is believed to be another way to effectively adjust their electronic conductance characteristics [92]. In general, it is difficult to precisely control the doping of hetero atoms into CNTs by conventional preparation techniques [93]. In addition, the N-doped multi-walled CNTs (MWCNTs) are usually bamboo-shaped, which makes it impossible to fill foreign matters into the hollow core of the N-doped CNTs [94,95]. Compared with the conventional synthesis methods [95-97], the AAO template approach shows unique advantage for 
hetero atom doping in CNTs [93,98-101]. The heteroatoms can be selectively doped at the inner or outer surface of CNTs, which is very useful in many applications requiring different polar nature and chemical or physical reaction activity, such as bioseparations and catalyst supports [14,39]. This unique advantage originates from the synthesis procedure of AAO-CNTs. The selective doping at the inner surface of AAO-CNTs is performed before the removal of the AAO template. Because the outer surface of the CNTs is embedded in the nanochannels of AAO template, the hetero dopant atoms are accessible only to the inner surface of the CNTs, thus the doping occurs only at the inner surface. The selective doping at the outer surface of CNTs is usually performed during the deposition of carbon onto the surface of the AAO template. Precursors containing carbon and hetero atoms are introduced simultaneously and then deposited onto the surface of the nanochannels, thus yielding outer surface doped CNTs. N and B are the most widely used hetero atoms for the doping of CNTs [102-105].

The process for preparing N-doped AAO-CNTs is schematically shown in Figure 10. Basically, three kinds of $\mathrm{N}$ doped AAO-CNTs can be obtained. The first is uniformly $\mathrm{N}$-doped CNTs, which is prepared by simultaneously carbon and nitrogen deposition onto the surface of AAO nanochannels using a $\mathrm{N}$-containing organic compound as $\mathrm{C} / \mathrm{N}$ source. The second is CNTs with $\mathrm{N}$ doped at the outer surface. In this case, a two-step CVD process is employed. A layer of $\mathrm{N}$-doped carbon is first deposited by using a $\mathrm{N}$ containing carbon source; then, the CVD of a pure carbon source yields the inner carbon layer. After removing the AAO template, CNTs with N selectively doped at the outer carbon layers are obtained. The third is CNTs with N selectively doped at the inner carbon layers, which is also obtained by a two-step CVD process, i.e. a pure carbon layer is firstly deposited and then the deposition of N-doped carbon layer. The amount of doped nitrogen can be tuned in the range of N/C atomic ratio $<0.1$ [98-101]. A unique feature of the N-doped CNTs synthesized by the AAO template method is that their hollow cores are empty, which is quite different from the bamboo-like structures of the N-doped CNTs by the conventional synthesis methods [95,106-108]. And such N-doped AAO-CNTs show advantage in the filling of foreign matters. Furthermore, the N-doping can be rather uniform for the AAO-CNTs.

Figure 11(a) shows the SEM image of a N-doped CNT sample prepared by the AAO template method using a Ncontaining organic compound as carbon source. The CNTs obtained are in form of well-aligned arrays. The length of the bundles is about $40 \mu \mathrm{m}$, exactly the same as the thickness of the AAO film template used. TEM observation

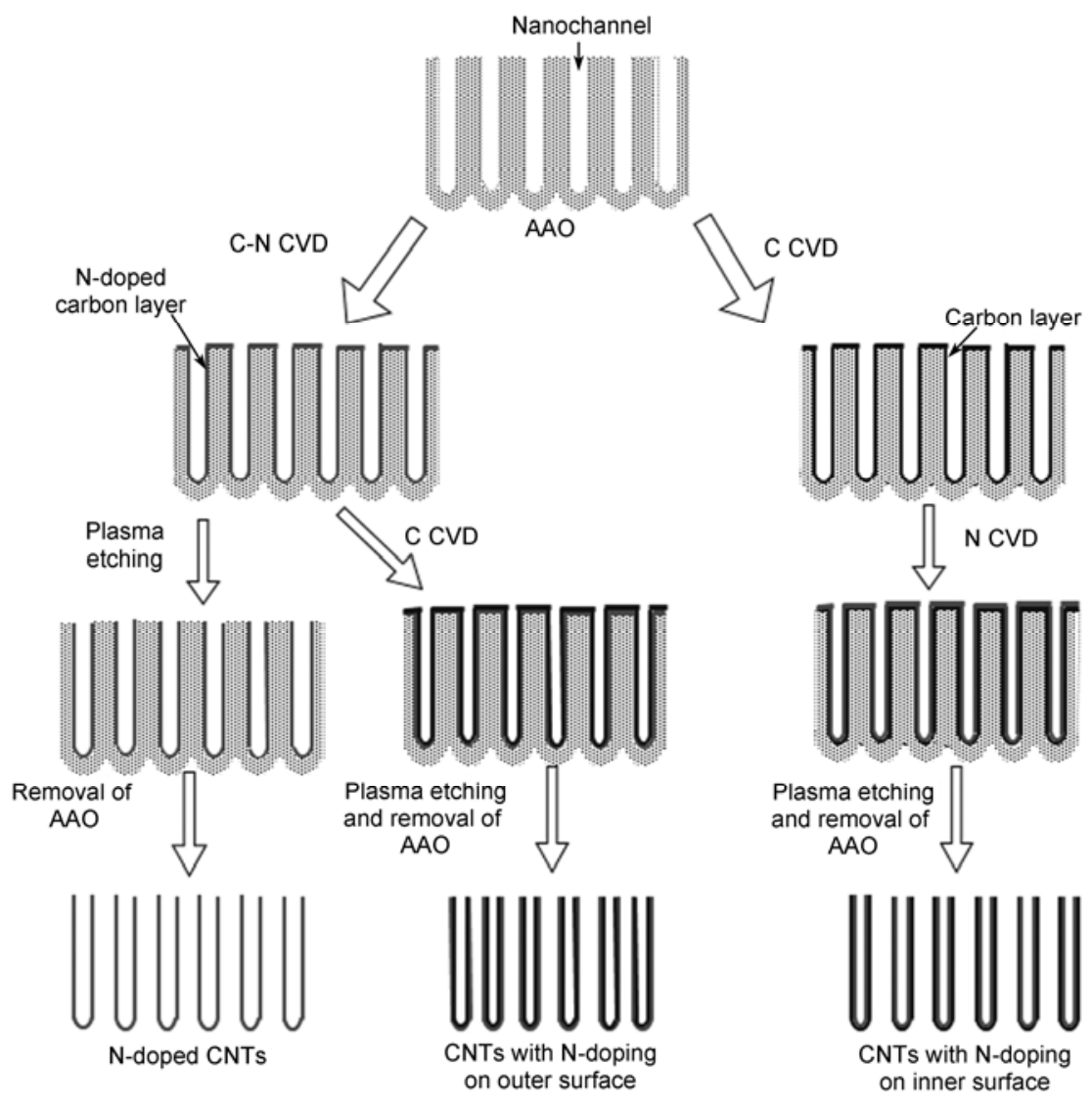

Figure 10 Schematic showing the preparation of N-doped CNTs with different doping sites. 

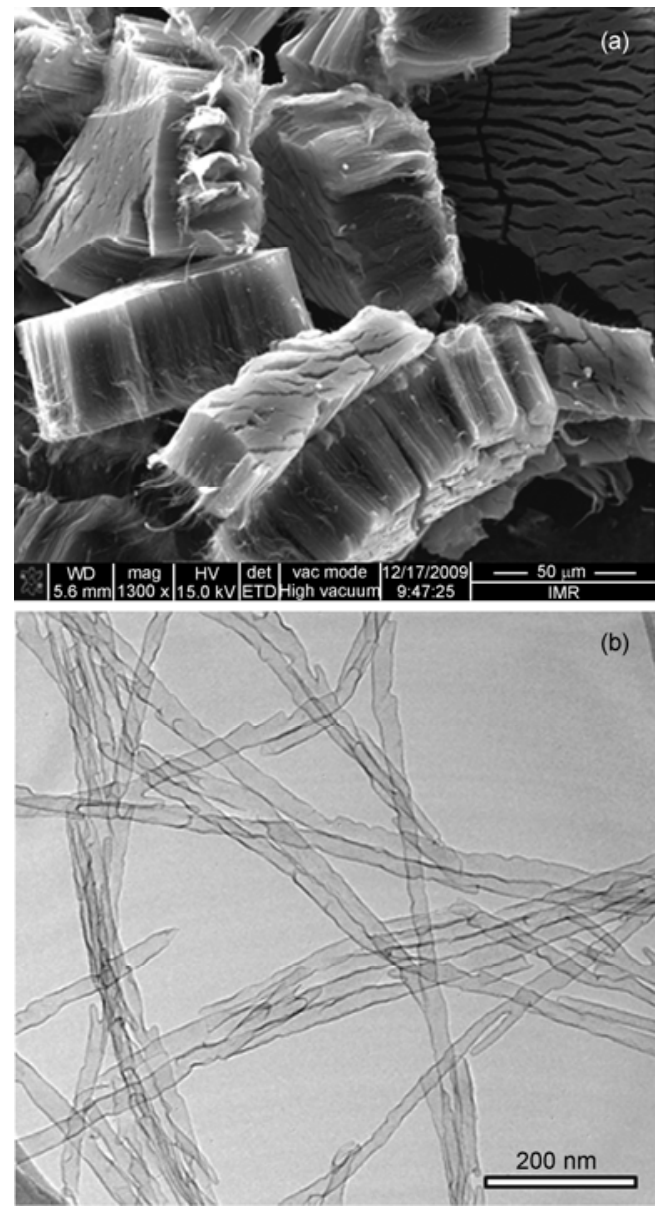

Figure 11 Typical SEM image (a) and TEM image (b) of the N-doped AAO-CNTs.

(Figure 11(b)) confirms that the outer diameter of the CNTs is about $20 \mathrm{~nm}$, which exactly replicates the internal diameter of the nanochannels of the AAO film. Moreover, it can be seen that the tube wall thickness is uniform along the tube axial direction, which implies that the deposition of carbon/nitrogen into the nanochannels of AAO template is uniform and continuous.

A SEM image of N-doped CNT array is shown in Figure 12(a), and the elemental distribution along the CNT axis direction is shown in Figure 12(b). It can be seen that the atomic percentage of $\mathrm{N}$ along the $\mathrm{CNT}$ axial direction is roughly a constant, which verifies the uniformity of the $\mathrm{N}$ doping. X-ray photoelectron spectroscopy analysis further confirms the successful doping of nitrogen in the CNTs, the spectra of N1s and C1s are shown in Figure 13(a) and (b), respectively. The N/C atomic ratio is calculated to be 0.09 , which is very high compared to the reported N-doped CNTs. The carbon layer of AAO-CNTs usually contain more structural defects (e.g. dangling and distorted bonds and functional groups) than for the CNTs by conventional techniques, which makes it easier for the doping of hetero atoms. In the N1s spectrum, the CNT exhibits a main peak at $401 \mathrm{eV}$ together with a small shoulder peak at $398 \mathrm{eV}$. The appearance
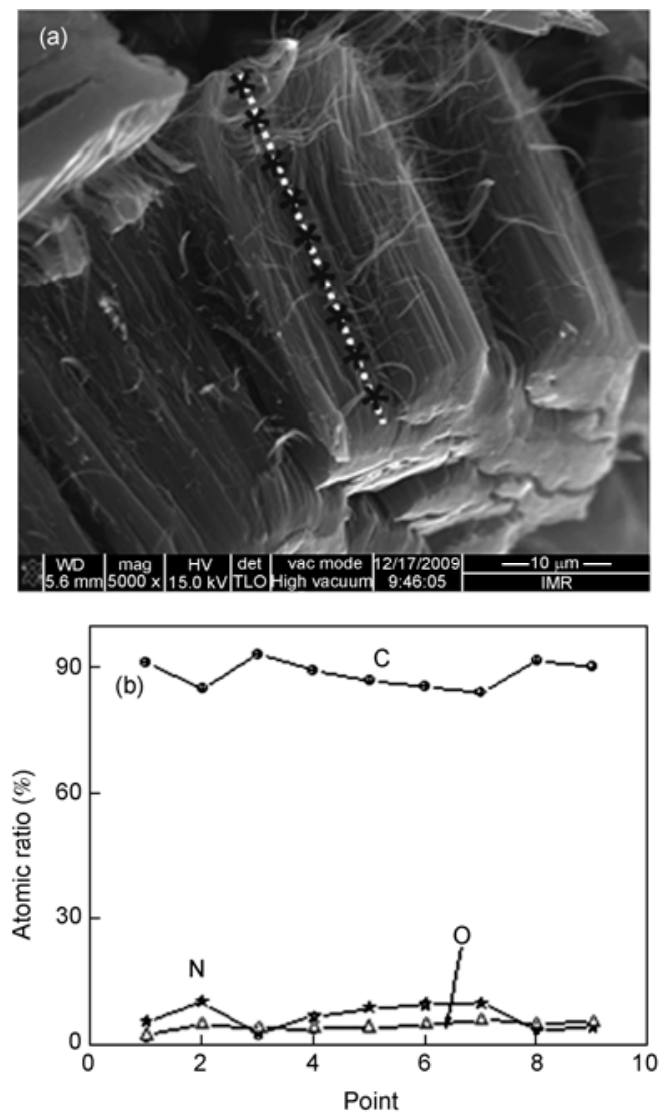

Figure 12 (a) SEM image of N-doped CNTs; (b) elemental distribution along the axis of the CNT array shown in (a) by energy dispersive X-ray spectrum (EDX) line scan.
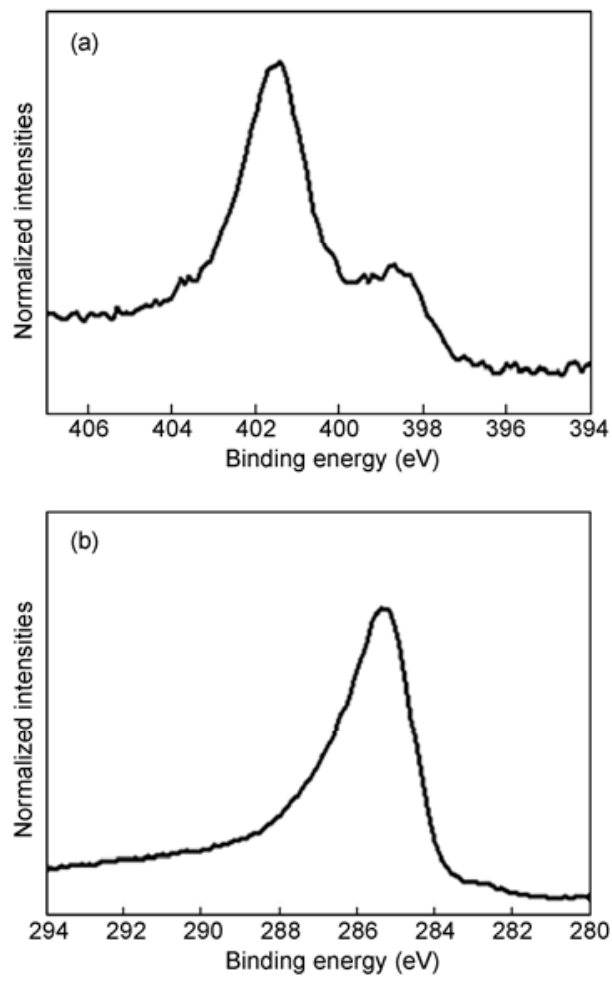

Figure 13 N1s (a) and C1s (b) XPS spectra of the N-doped AAO-CNTs. 
of these two peaks suggests the presence of quaternary and pyridine-type nitrogen species, respectively. A peak centered at around $285 \mathrm{eV}$ is observed in the $\mathrm{C} 1 \mathrm{~s}$ spectrum, which can be attributed to the $\mathrm{sp}^{2}$ carbon atoms of the carbon skeleton.

Therefore, $\mathrm{N}$ can be easily doped into the carbon skeleton of AAO-CNTs, and the uniformly doped nitrogen mainly exists as quaternary and pyridine type. The N-doped AAOCNTs have empty hollow cores rather than bamboo-like structure, which facilitate the filling of foreign matters inside their hollow cores.

Different from the controllable doping site for nitrogen, boron can be only doped at the inner surface of AAO-CNTs, because it is very difficult to co-deposit carbon and boron onto the surface of AAO nanochannels. However, once a layer of pure carbon or $\mathrm{N}$-doped carbon has been firstly deposited onto the surface of AAO nanochannels, B-containing carbon layers can be easily further deposited. Therefore, two types of B-doped CNTs can be obtained. Type one is CNTs with pure carbon outer layer and B-doped carbon inner layer. Type two is CNTs with N-doped carbon outer layer and B-doped carbon inner layer. The content of doped boron can be controlled in the $\mathrm{B} / \mathrm{C}$ atomic ratio range of 0-0.07 [99]. Similar with N-doping, the high content Bdoping can be attributed to the defects-rich tube wall of the AAO-CNTs.

TEM images of a N-doped CNT and a coaxial N, Bdoped CNT are shown in Figure 14. These two types of CNTs were synthesized by one-step and two-step CVD process, respectively. It is apparent that both of the CNTs have uniform carbon layers (N-doped CNT: $1.5 \mathrm{~nm}$; N, Bdoped CNT: $5.0 \mathrm{~nm}$ ). In other words, the N, B-CNTs have coaxial carbon layers, the outer layers (1.5 $\mathrm{nm}$ of thickness, about 4 graphene sheets) and the inner layers $(3.5 \mathrm{~nm}$, about 10 graphene sheets) were prepared by the first and second step of CVD, respectively. HRTEM image (the inset of Figure 14(b)) reveals that there is no visible difference between the outer and inner layers prepared by the different CVD steps (4N-doped and 10B-doped layers are roughly distinguished from each other by the dotted line). The surface composition of inner and outer layers of the N, Bdoped CNTs was characterized using XPS. Generally, an XPS spectrum mainly reflects the information of the outer layer of the measured specimen, and it is difficult to detect its inner layer. However, in the case of AAO-CNTs, the characteristics of their inner surface (inner layer) can be estimated from the analyses of the external surface (upper layer) of the corresponding carbon-coated AAO [101]. The XPS spectrum of the carbon-coated AAO film are regarded as that of the inner surface (inner layer) of the resulting nanotubes, which is also a unique characteristic of AAOCNTs. The XPS spectra of inner and outer layer of the N, B-doped CNTs are shown in Figure 15. It can be seen that carbon-coated AAO (inner layer of resulting NB-CNTs) are characterized by a large B1s peak without any N1s peaks,
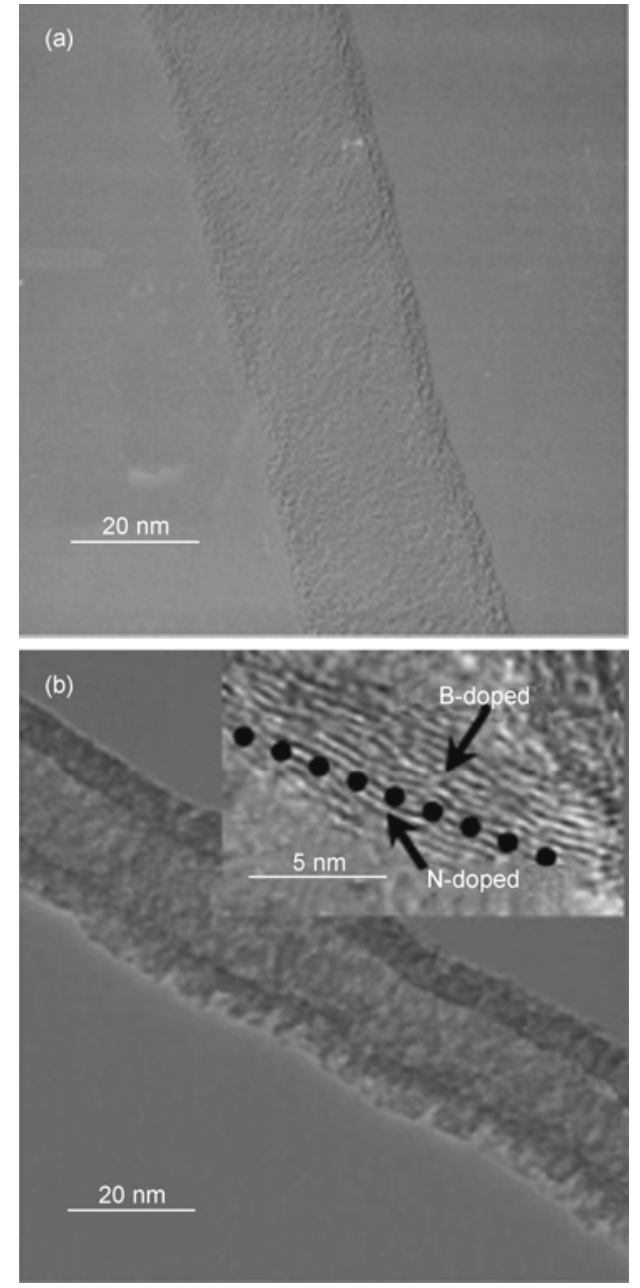

Figure 14 TEM images of (a) an N-doped CNT and (b) an N, B-doped CNT. Inset of (b): HRTEM image of the wall of an N, B-doped CNT [100].

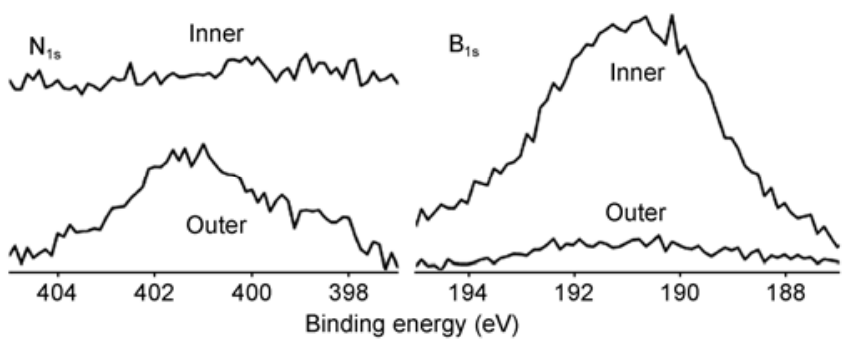

Figure $15 \mathrm{~N} 1 \mathrm{~s}$ and B1s XPS spectra of the N, B-doped CNT and the corresponding AAO employed [100].

while the resulting nanotubes, N, B-doped CNTs, possess a sound N1s peak with a very small B1s peak. It is thus easy to conclude that the N, B-doped CNTs have coaxial sidewall structure of outer $\mathrm{N}$-doped (N/C atomic ratio: 0.025$)$ and inner B-doped layers (B/C: 0.040).

\section{Filling of AAO-CNTs}

CNTs have unique tubular structure and nanoscale hollow 
cores. The diameter of the nanochannels can be tuned in the range of $1-100 \mathrm{~nm}$, and the length of the CNTs is also controllable. Therefore, CNTs provide nanoscale space for loading foreign matters inside their hollow cores. The filled materials may show intriguing properties $[43,109,110]$ due to the space confinement effect and the interactions with the surrounded carbon layers. On the other hand, the nanochannel of CNTs provides ideal delivery vehicles for the payloads of biomolecules, drugs, DNA, and metals [39]. Furthermore, the hollow core of CNTs can also act as an ideal nanoreactor for gas or liquid reactions [11].

Many studies concerning the opening, cutting, and filling of the CNTs synthesized using conventional synthesis method have been conducted [111-115]. A knotty problem is that foreign maters may deposit onto the outer surface of CNTs during the filling procedure by using either incipient wetness impregnation method or sublimation method $[116,117]$. Therefore, the removal of foreign materials from the outer surface of CNTs is required. On the other hand, a complete filling of foreign matters inside the hollow core of CNTs can be achieved by using the AAO template method. This unique advantage originates from the preparation and filling process of the AAO-CNTs.

The flow chart of filling foreign matters into AAO-CNTs is shown in Figure 16. The AAO-CNTs used can be either one-side open or both-side open. Foreign matters can be loaded into the carbon-deposited AAO template by using incipient wetness impregnation [40,42,118-121], sublimation [122-127], or electrodeposition method [41,109,128131]. Since only the inner surface of the AAO-CNTs is exposed, the foreign matters would be selectively filled inside the hollow cores of CNTs. After the filling, the AAO template is dissolved by immersing it in $\mathrm{NaOH}$ or aicd aqueous solution. As a result, CNTs with foreign matters selectively filled inside the hollow cores are obtained. The main techniques used for the filling of AAO-CNTs, i.e. incipient wetness impregnation, sublimation, and electrodeposition will be introduced as follows.

\subsection{Wetness impregnation technique}

Wetness impregnation is a commonly used method for filling foreign matters into the hollow core of CNTs [132]. The precursor used in the wetness impregnation method is usually a soluble salt that contains the element intended to fill. After the impregnation of the salt solution, reduction or oxidation process are often used. Kyotani et al. [120,121] first reported the filling of Pt and Ag into AAO-CNTs. Later, Che et al. [42] realized the filling of $\mathrm{Pt} / \mathrm{Ru}$ nanoparticles. Gogotsi et al. directly filled $\mathrm{CuO}$ particles with a diameter of $\sim 5 \mathrm{~nm}$ into the cavity of AAO-CNTs with an outer diameter of $200 \mathrm{~nm}$ [119]. We will next give an example of the filling of $\mathrm{Fe}_{2} \mathrm{O}_{3}$ nanoparticles into the hollow core of AAOCNTs by using the incipient wetness impregnation technique, where the selectivity, homogeneity, and controllability of the filling are clearly shown [118].

Figure 17 shows SEM images and the corresponding EDX spectra of the AAO-CNTs before (a) and after (b) the filling of $\mathrm{Fe}_{2} \mathrm{O}_{3}$ nanoparticles. $\mathrm{No} \mathrm{Fe}_{2} \mathrm{O}_{3}$ nano-particles or impurities can be observed on the outer surface of the CNTs, indicating that all the $\mathrm{Fe}_{2} \mathrm{O}_{3}$ nano-particles are successfully loaded into the hollow core of the CNTs. Under SEM, the CNTs with and without $\mathrm{Fe}_{2} \mathrm{O}_{3}$ filling basically show similar morphology, except for a difference in contrast. The EDX spectrum of the CNTs is shown in Figure 17(c) and (d). Only carbon and oxygen can be detected in the pristine sample. However, iron signal is clearly seen for the filled CNTs.

TEM observations show (Figure 18(a) and (b)) that the $\mathrm{Fe}_{2} \mathrm{O}_{3}$ nano-particles are homogeneously filled in the CNTs, and there is not any impurity attached on the outer surface of the CNTs. The filled $\mathrm{Fe}_{2} \mathrm{O}_{3}$ particles are uniform in size with a mean diameter of $\sim 9 \mathrm{~nm}$. The diameters of around $100 \mathrm{Fe}_{2} \mathrm{O}_{3}$ particles were measured and the resultant histogram of the particle size distribution is plotted in Figure 18(c). It can be seen that the diameters of the nanoparticles range from 4 to $14 \mathrm{~nm}$ with a mean value of $9.3 \mathrm{~nm}$.

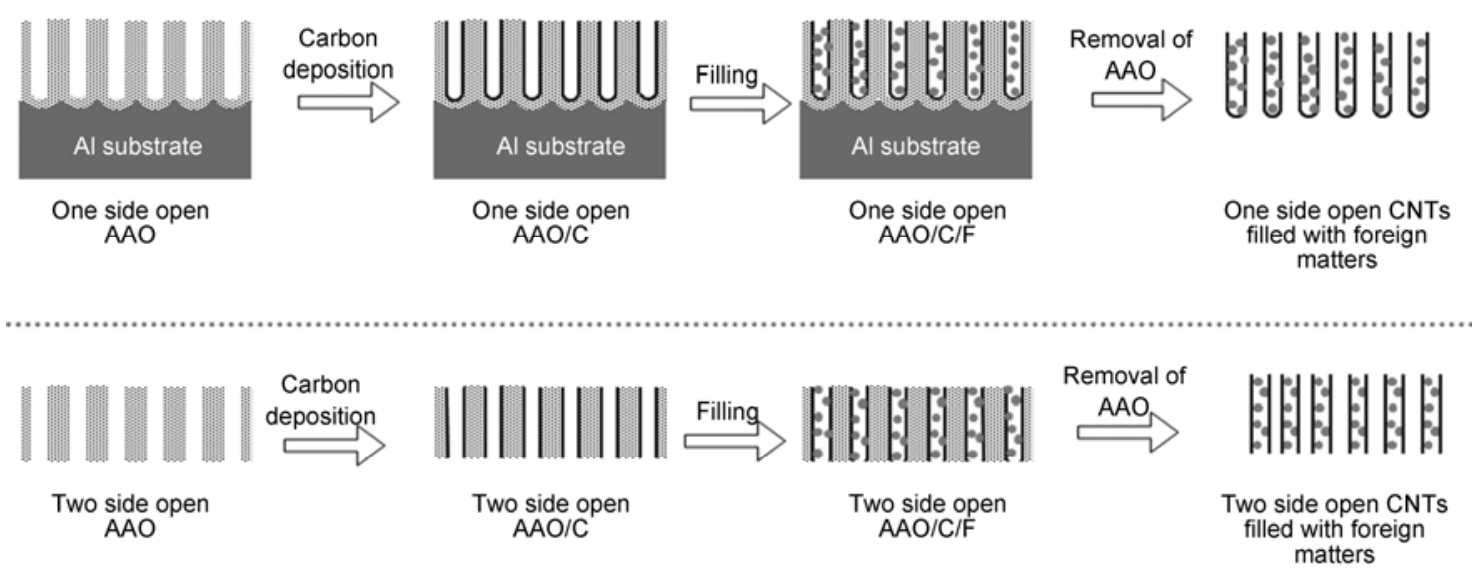

Figure 16 Schematic diagram showing the selective filling of foreign matters into the hollow core of AAO-CNTs. 

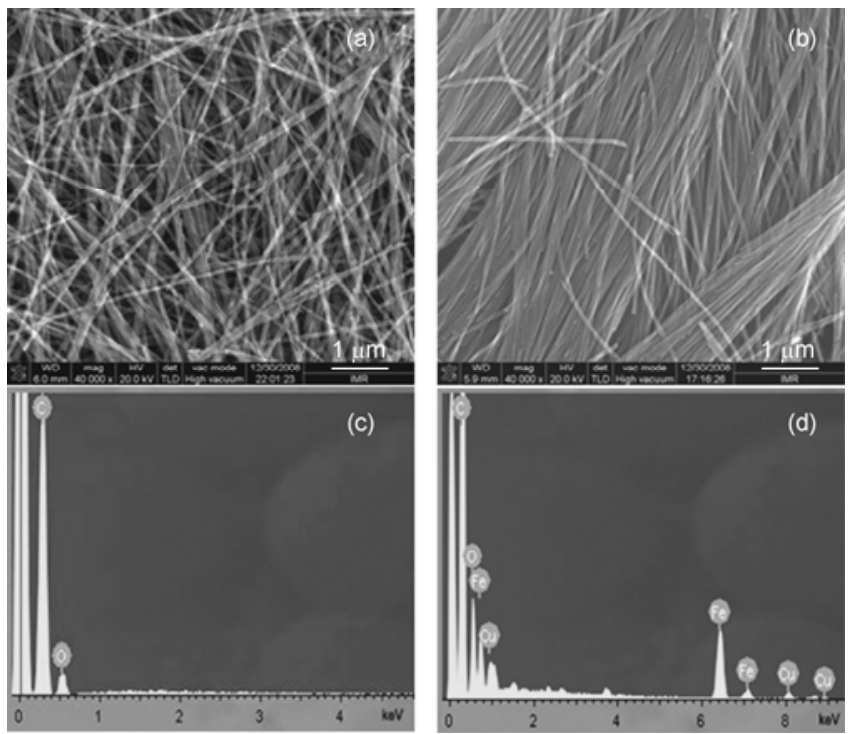

Figure 17 SEM images of the CNTs before (a) and after (b) $\mathrm{Fe}_{2} \mathrm{O}_{3}$ filling, and the corresponding EDX spectra (c), (d) [118].

In addition, the filling ratio of the $\mathrm{Fe}_{2} \mathrm{O}_{3}$ nanoparticles can be easily controlled by changing the concentration of iron nitrate solution used during the filling process. When the concentration of iron nitrate solution increased from $2 \mathrm{wt} \%$ to $25 \mathrm{wt} \%$, the $\mathrm{Fe}_{2} \mathrm{O}_{3}$ contents are increased from $33 \mathrm{wt} \%$ to $60 \mathrm{wt} \%$. At the same time, the homogeneity of the filled $\mathrm{Fe}_{2} \mathrm{O}_{3}$ particles can be well retained.

\subsection{Sublimation method}

Sublimation of a metal, metal precursor, or metal oxide precursor are also used to load foreign matters into the cavity of CNTs [122-124]. Basically, all foreign matters that can be sublimated at low temperature are adaptable to this technique. However, the material filled into the AAO-CNTs should be stable during the subsequent removal of AAO template.

Iron oxide particle was reported to be filled into the cavity of AAO-CNTs using a metal-organic chemical vapor deposition (MOCVD) technique $[125,126]$. The iron precursor used is ferrocene, and the filling process includes the vaporization of ferrocene and Fe chemical vapor deposition. First, ferrocene was vaporized at low temperature (90 or $\left.105^{\circ} \mathrm{C}\right)$. Then, the vapor was introduced into the carbon coated AAO film to deposit Fe into the nanochannels. After the MOCVD process, the film was treated with $\mathrm{NaOH}$ solution to remove the anodic aluminum oxide template. As a result, CNTs filled with metal oxide nanoparticles were obtained as an insoluble fraction. Furthermore, the filling amount and particle size can be controlled by changing the MOCVD conditions. It is worthy to note that the conversion of iron to iron oxide takes place when the Fe/C/AAO is exposed to air and during the removal of the AAO template, because of the high reactivity of the nanometer sized iron paticles. By using the MOCVD technique, nickel nanowires with a diameter of $4 \mathrm{~nm}$ were also filled into the cavity of CNTs with an inner diameter of $20 \mathrm{~nm}$ using cobaltocene as precursor [127]. Therefore, nanowires and nanoparticles can be selectively filled in CNTs using the sublimation method.

\subsection{Electrodeposition}

Metal/metal alloy can also be filled into the nanochannels of AAO-CNTs using a conventional electrodeposition technique. When an electric field is applied, metal ions in the electrolyte solution migrate and are deposited onto an electrode by passing a current through an electrochemical cell. Therefore, metals can be filled into the cavity of AAOCNTs by using the electrodeposition method under a direct current $[41,128,129]$ or alternating current [106]. In general, a layer of $\mathrm{Au}$ is first deposited on the surface of a both-side open AAO film using ion sputtering technique. Then, metal foreigner can be deposited into the hollow core of AAOCNTs by using the pre-deposited Au layer and graphite plate as working-electrode and counter-electrode, respectively. However, it is rather difficult to handle during the electrodepositon process owing to the fragile property of AAO films. Even though using the AAO film together with the $\mathrm{Al}$ support as an electrode can overcome this problem,
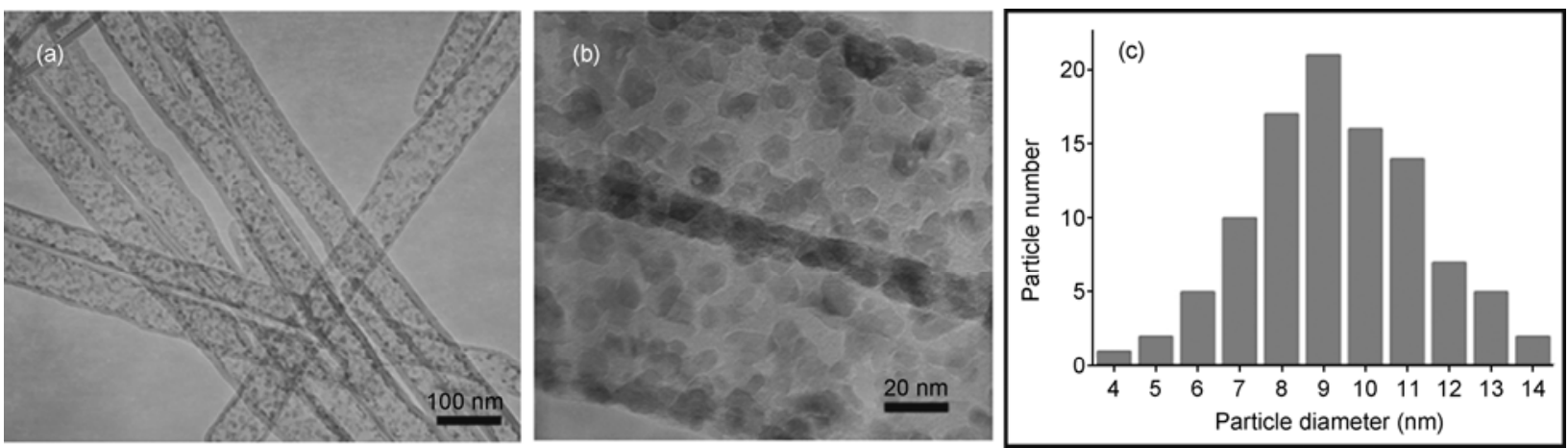

Figure 18 (a) Low- and (b) high-magnification TEM images of the $\mathrm{Fe}_{2} \mathrm{O}_{3}$-filled CNTs; (c) a histogram showing the size distribution of the $\mathrm{Fe}_{2} \mathrm{O}_{3}$ particles [118]. 
the barrier layer existing at the pore bottom makes the AAO pores have a closed end which insulates it from the Al substrate.

Wang et al. [41] first filled Permalloy into a one-endopened CNT embedded in an AAO film connected with Al substrate. In spite of the conductive outer carbon film, it was unambiguously demonstrated that the Permalloy filling started from the pore bottoms of CNTs. In addition, the filled materials can be in forms of either particles or rods (Figure 19). This breakthrough is mainly originated from high minus voltage, mild oxidation in $\mathrm{H}_{2} \mathrm{O}_{2}$, and thin barrier layer owing to the process of widening nanochannels. This novel bottom-up filling opens a route to controllable filling of materials of interest into CNTs with one open end. Recently, aligned Ni nanoparticles array are also reported to be encapsulated in the channel of AAO-CNT using alternating current by decreasing the thickness of the barrier layer [106]. At the same time, magnetic metal or alloy filled in the cavity of CNTs is responsive to magnetic field even after a long-term storage in water. Furthermore, the metal-filled CNTs can be water dispersible after a $\mathrm{H}_{2} \mathrm{O}_{2}$ treatment [128].

In general, filling foreign matters into the hollow core of AAO-CNTs takes place after the deposition of carbon layer onto AAO film. Recently, we altered the experimental order, and Pt-filled CNTs were obtained [133]. In a typical experiment, approximately $200 \mathrm{~nm}$ thick Ag layer was deposited onto the obtained AAO film as a conductive layer by sputtering. Pt nanowires were then electrodeposited into the nano-channels of the AAO template by three-electrode direct current electro-deposition in an electrolyte containing $\mathrm{H}_{2} \mathrm{PtCl}_{6}$ and boric acid. After the electro-deposition of $\mathrm{Pt}$ into the nano-channels of the AAO template, the as-obtained sample was placed in a vertical quartz furnace for chemical vapor deposition of carbon. The resulted materials were immersed into a $5 \mathrm{~mol} / \mathrm{L} \mathrm{NaOH}$ aqueous solution to remove the AAO template. Finally, Pt-filled CNTs were obtained as shown in Figure 20. We design and fabricate CNT-clamped Pt atomic chains (as shown in Figure 21), which can provide a general approach for the interconnection and integration of metal atomic chains with CNTs. The strategy we proposed is effective in fabricating a variety of metal atomic chains and in situ connecting these metal atomic chains with CNTs, thus it may find potential applications in the assembly of nano/subnano devices.

In summary, the filling of foreign matters into AAOCNTs can be achieved by using wetness impregnation, sublimation and electrodeposition techniques. Compared with the cut and opened CNTs synthesized by conventional methods, an obvious advantage of the AAO-CNTs is that $100 \%$ filling of foreign matters inside the cavity of CNTs can be easily achieved, and the content, morphology, and homogeneity of the filled materials can be well controlled.

\section{Challenge and developing trends of AAO-CNTs}

As described above, the AAO-CNTs have many unique advantages in controllability, uniformity, heteroatom doping,
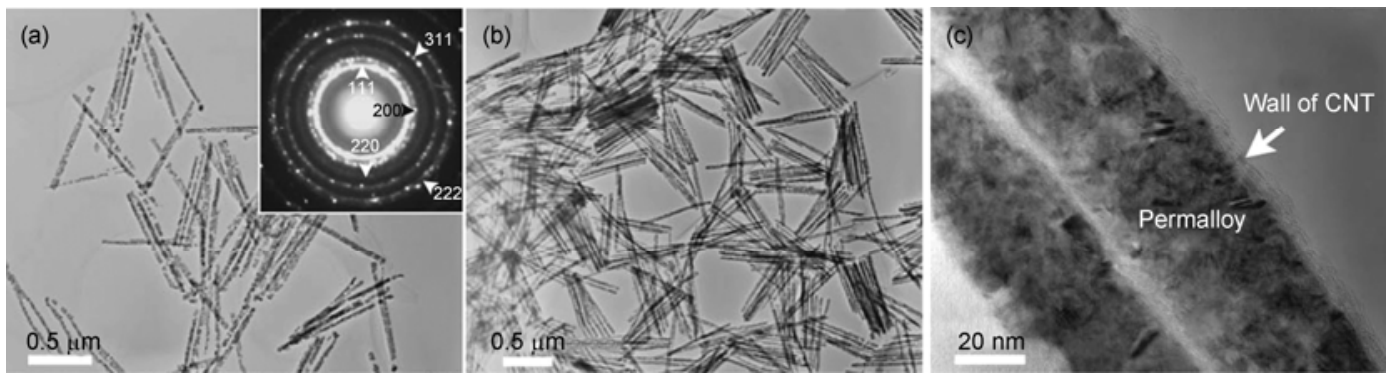

Figure 19 TEM images of Permalloy-filled CNTs by (a) voltage-stepped electrochemical deposition and (b) pulsed electrochemical deposition, followed by oxygen plasma etching; (c) a high-magnification image of the sample observed in (b), showing that the Permalloy is still retained in the nanotubes [41].
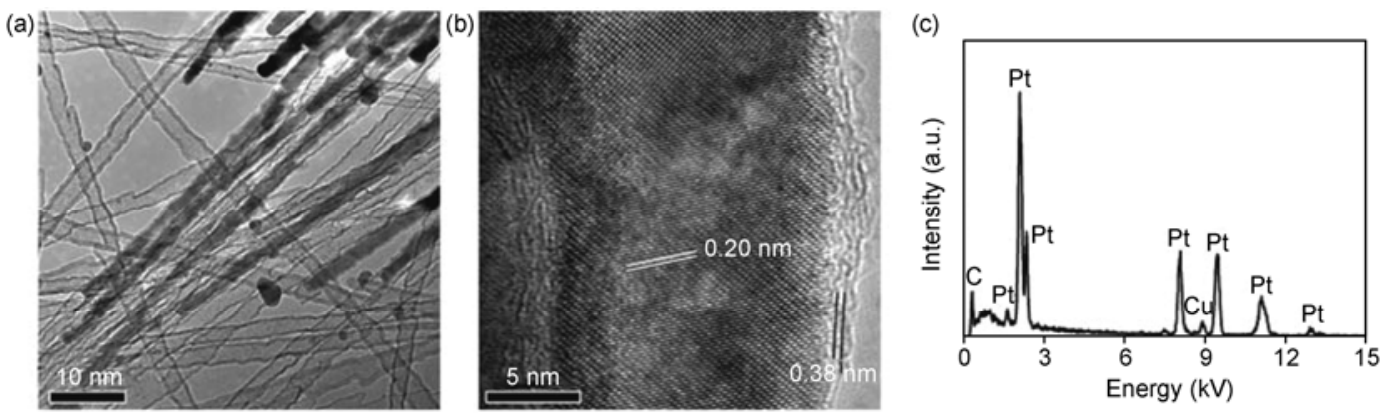

Figure 20 TEM image (a), HRTEM image (b), and energy disperse spectrum (EDS) (c) of the Pt-filled CNTs prepared by an AAO template method. The diameter of the CNTs is about $30 \mathrm{~nm}$. Lattices corresponding to Pt (200) and carbon layers are clearly resolved. The composition is confirmed to be Pt and C by EDS analysis (the $\mathrm{Cu}$ signal is from $\mathrm{Cu}$ grid) [133]. 

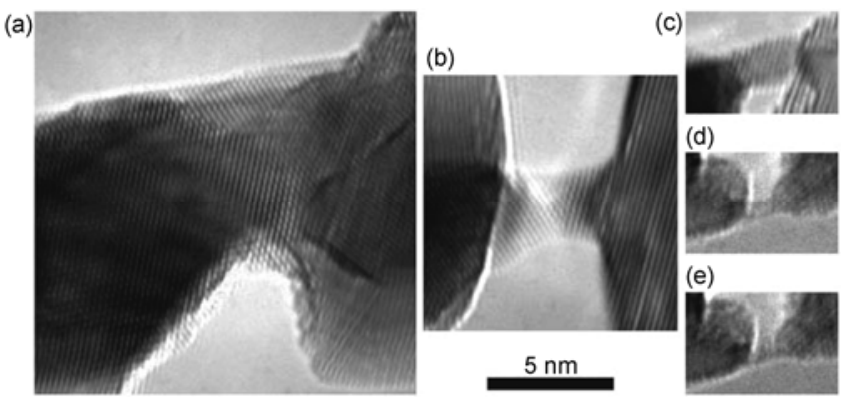

Figure 21 HRTEM images showing the formation process of a CNTclamped Pt atomic chain. (a) and (b) Electron beam is focused on the exposed Pt nanorod after carbon layers are removed by electron irradiation; (c)-(e) the formation of a Pt atomic chain is observed [133].

and filling foreign matters. However, there are also shortcomings for the AAO-CNTs, which may limit their further development, such as low crystallinity, high cost, and inaccessibility of small diameter CNTs.

\subsection{Poor crystallinity of AAO-CNTs}

The carbon layers of AAO-CNTs are usually wavy and discontinuous (Figure 22(a)), when compared with the CNTs synthesized by conventional method (Figure 22(b)) [134]. To improve the crystallinity of AAO-CNTs, performing
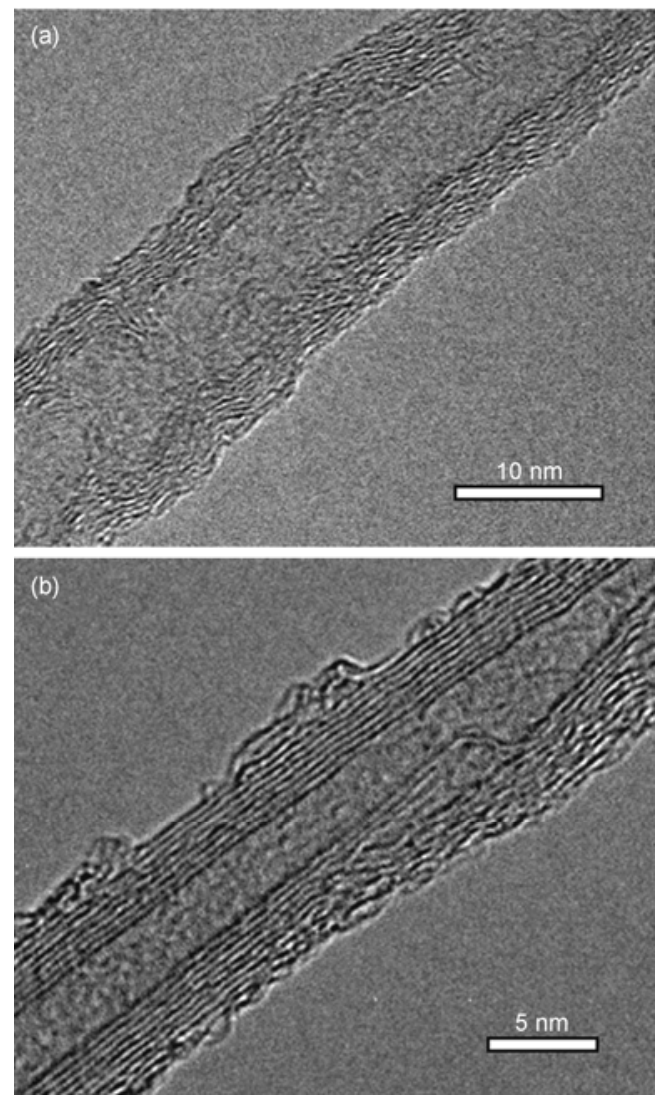

Figure 22 HRTEM images of a typical AAO-CNT (a), and a CNT synthesized by the conventional CVD method (b).
CVD process at higher temperatures is a generally used method [99]. It was found that the crystallinity can be improved when the CVD temperature was increased from 500 to $800^{\circ} \mathrm{C}$, but no obvious difference was observed when the temperature was further improved to $1000^{\circ} \mathrm{C}$ [135].

An alternative method is to perform heat treatment or graphitization treatment after the AAO-CNTs are liberated from AAO template. Beguin et al. [136,137] first investigated the effect of heat treatment on the structure of AAOCNTs. They reported that the crystalline structure of the AAO-CNTs could be improved by annealing at elevated temperatures between 1600 and $2400^{\circ} \mathrm{C}$ under argon atmosphere. Especially, remarkable changes on the morphology and development of a highly crystalline structure were observed for the CNTs annealed at $2400^{\circ} \mathrm{C}$. However, the high temperature treatment usually creates some impurities of carbon fragments, which are not desired.

We performed heat-treatment of AAO-CNTs at $1450^{\circ} \mathrm{C}$. It can be seen that the crystallinity of the CNTs is improved (Figure 23(a)). The Raman spectra of a CVD-CNT sample and the AAO-CNTs before and after $1450^{\circ} \mathrm{C}$ treatment are shown in Figure 23(b). We can see that the G/D intensity ratio of the heat-treated AAO-CNTs is obviously increased and turns similar to that of CVD-CNTs. Thermal analysis of the AAO-CNTs before and after heat treatment was performed in air atmosphere (Figure 23(c)). It was found that the starting oxidation temperature is increased from $~ 300$ to $\sim 550^{\circ} \mathrm{C}$ after the heat treatment. XPS and cryo-nitrogen adsorption characterizations (Table 1) suggested that, the oxygen content and BET surface area of the heat treated AAO-CNTs are decreased, and their pore size is slightly increased. Therefore, the crystallinity of the AAO-CNT can be improved through high temperature treatments, during which, oxygen-containing functional groups can be removed, structural defects are healed, and the carbon layers turn more straight and regular.

Although heat treatment is feasible and effective in improving the crystallinity of AAO-CNTs, there are also limitations for this approach. For example, it can not be applied to foreign matters-filled AAO-CNTs, because the filled matters may be vaporized due to the high temperature. In addition, the heat treatment may also introduce some undesired carbon fragments and hence lead to a degraded property of the AAO-CNTs. There are also some woks on the growth of CNTs from metal catalyst pre-deposited into the channels of AAO film [138-142]. CNTs thus obtained have good crystallinity. However, the deposition process is complex, and the uniformatin of the CNTs can not be ensured. Therefore, it is highly desired to establish appropriate approach and conditions for synthesizing high quality AAOCNTs directly.

\subsection{Obtaining AAO-CNTs with smaller diameter}

The smallest outer diameter of AAO-CNTs was reported to 

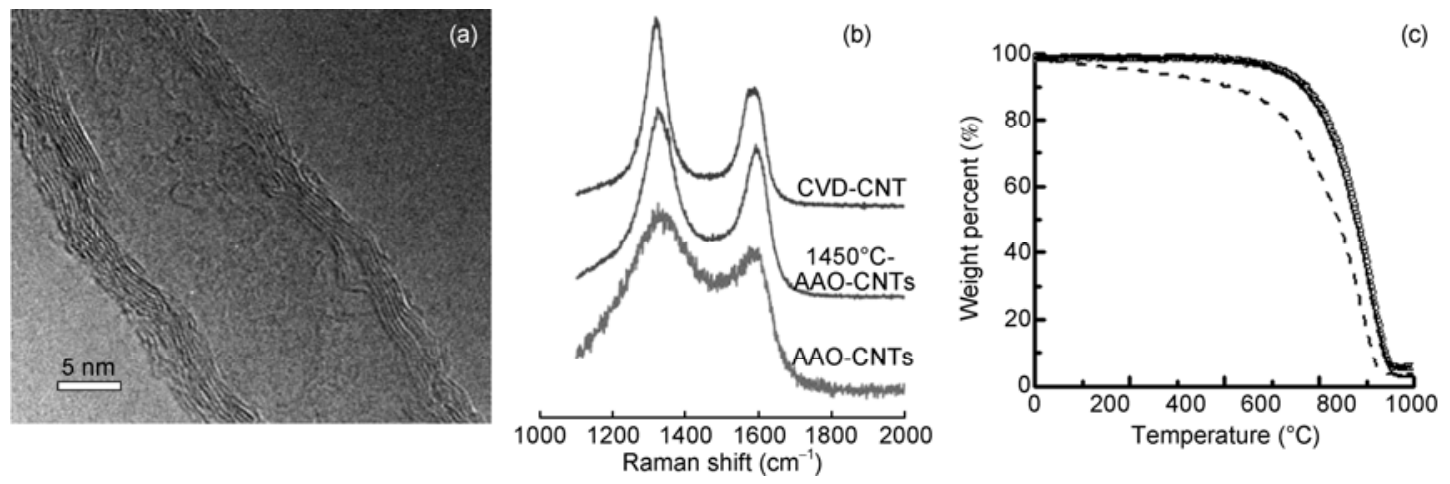

Figure 23 (a) HRTEM image of the AAO-CNTs heat treated at $1450^{\circ} \mathrm{C}$; (b) Raman spectra of the AAO-CNTs before and after $1450^{\circ} \mathrm{C}$ treatment, and that of CVD-CNTs for comparison; (c) TG curves of AAO-CNTs (dash line), AAO-CNTs after $1450^{\circ} \mathrm{C}$ treatment (solid line), and CVD-CNTs (circle line).

Table $1 \mathrm{O} / \mathrm{C}$ atomic ratio and BET surface area of the AAO-CNTs before and after the heat treatment

\begin{tabular}{lccc}
\hline & $\mathrm{O} / \mathrm{C}($ atomic ratio \%) & $S_{\mathrm{BET}}\left(\mathrm{m}^{2} / \mathrm{g}\right)$ & Pore size $(\mathrm{nm})$ \\
\hline AAO-CNTs & 14.5 & 613 & 15.5 \\
$1450^{\circ} \mathrm{C}$-treatment & 9.8 & 462 & 16.1 \\
\hline
\end{tabular}

be $\sim 10 \mathrm{~nm}$ [76]. Even smaller CNTs are required for some applications such as filter membrane, catalyst supports, and electronic devices. In addition, the CNTs with smaller diameters are more attractable for investigating their confinement effect $[10,12,13,143]$.

To obtain AAO-CNTs with smaller outer diameter, the key issue is to prepare AAO template with smaller nanochannel size. The smallest pores formed by conventional anodizing have a diameter of $\sim 7 \mathrm{~nm}$ [144]. Recently, Inada et al. [145] developed a novel anodizing technique which employed a pulse sequential voltage with a frequency of $100 \mathrm{~Hz}$. Low anodizing potentials (below $3 \mathrm{~V}$ ) were used, and the linear dependence between pore diameter and voltage does not exist in this case. However, pores with diameters of 3 and $4 \mathrm{~nm}$ can be successfully formed at pulse sequential voltages of 1 and $2 \mathrm{~V}$, respectively.

Although some progresses have been made, systematic studies are still needed to develop efficient techniques and to elucidate the principle for preparing AAO template with small diameters, which is a precondition to obtain small diameter, or even single-walled AAO-CNTs.

\subsection{High cost and low synthesis efficiency}

An obvious limitation of the AAO template method is the low synthesis efficiency. The preparation of the AAO-template and followed CVD of carbon deposition and removal of AAO template are complex and time-consuming [146]. The above procedure determines that the yield of AAOCNTs is limited, and the low utilization efficiency of the AAO template increases the cost of AAO-CNTs. Therefore, it seems not possible that the AAO-template technique can be used for mass production of CNTs. However, due to their unique preparation procedure and structural characteristics, the AAO-CNTs can be ideal model material for investigating novel physical and chemical properties of filled maters that originate from the confinement effect of CNTs. And some specific applications requiring precise structural control of CNTs can also be expected for the AAO-CNTs.

\subsection{Other limitations}

As described above, the AAO-CNTs can be either one side open or both-side open. While it is difficult to synthesize two-side close AAO-CNTs. Beguin et al. [137] reported that the open tips of AAO-CNTs can be closed after high temperature treatment. However, this process is not applicable for the heteroatom doped and foreign matters filled CNTs. The length of CNTs is also an important factor influencing their properties. Orikasa et al. [76] reported that carbon nano test tubes (one side open CNTs) with lengths of $5 \mu \mathrm{m}$ or less were soluble in water without further modification. More importantly, short length CNTs are preferred in some practical applications, such as catalyst supports [143], biological imaging [146], molecular sensing [147], etc. It is easy to obtain short nano test tubes using AAO template method, and the shortest one is $\sim 200 \mathrm{~nm}$. However, it is difficult to obtain short AAO-CNTs with good crystallinity owing to the bottlenecks on obtaining free-standing AAO template with thin thickness. Therefore, to realize the precise control over the diameter, cap-structure, and length of AAO-CNTs, it is important to develop efficient techniques that enable the preparation of AAO-template with desired structures.

\section{Conclusions}

AAO template method is very useful for the preparation of CNTs with specific and controllable shape, length, diameter, and wall thickness. Selective heteroatom doping at the outer or inner carbon layers of CNTs can also be realized for 
AAO-CNTs. In addition, with the aid of the AAO template, $100 \%$ filling of foreign matters into the hollow core of CNTs can be easily achieved. Except for the above unique characters, excellent uniformity and reproducibility are obvious advantages of AAO-CNTs in comparison to the CNTs by conventional synthesis methods. On the other hand, the shortcomings of low efficiency and high cost make it difficult to realize mass production of CNTs by the AAO-template method. Therefore, AAO-CNTs can be ideal model material for investigating the nanosize effect of CNTs, and to explore the physical and chemical properties of foreign matters filled inside the nano test tube. In addition, the AAO-CNTs may also find applications in some specific areas such as drug delivery, magnetic delivery carrier, catalystsupport, electronic device, biosensor, etc. With improvement on the control of the crystallinity, diameter, and cap structure, the function and application of AAO-CNTs are hopefully to be expanded further.

This work was supported by the Ministry of Science and Technology of China (2011CB932601, 2011CB932604, and 2008DFA51400), the Clean Energy Facing the Future Program between the Chinese Academy of Sciences and British Petrleum, and National Natural Science Foundation of China (50921004 and 50872137) and Scientific Research Foundation for the Returned Overseas Chinese Scholars, State Education Ministry.

1 Abbaslou R M M, Tavasoli A, Dalai A K. Effect of pre-treatment on physico-chemical properites and stability of carbon nanotubes supported iron fischer-tropsch catalysts. Appl Catal A-Gen, 2009, 335: 33-41

2 Tavasoli A, Abbaslou R M M, Trepanier M, et al. Fischer-tropsch synthesis over cobalt catalyst supported on carbon nanotubes in a slurry reactor. Appl Catal A-Gen, 2008, 345: 134-142

3 Chen W, Pan X L, Bao X H. Tuning of redox properties of iron and iron oxides via encapsulation within carbon nanotubes. J Am Chem Soc, 2007, 129: 7421-7426

4 Zhang H B, Pan X L, Liu J Y, et al. Enhanced catalytic activity of sub-nanometer titania clusters confined inside double-wall carbon nanotubes. ChemSusChem, 2011, 4: 975-980

5 Bahome M C, Jewell L L, Padayachy K D, et al. Fe-Ru small particle bimetallic catalysts supported on carbon nanotubes for use in fischer-Tropsch synthesis. Appl Catal A-Gen, 2007, 328: 243-251

6 Ma W, Kugler E L, Wright J, et al. Mo-Fe catalysts supported on activated carbon for synthesis of liquid fuels by the Fischer-Tropsch process: Effect of Mo addition on reducibility, activity, and hydrocarbon selectivity. Energy Fuels, 2006, 20: 2299-2307

7 Serp P, Corrias M, Kalck P. Carbon nanotubes and nanofibers in catalysis. Appl Catal A-Gen, 2003, 253: 337-358

8 Meng $\mathrm{C} \mathrm{Z}$, Liu C H, Chen L Z, et al. Highly flexible and all-solidstate paper like polymer supercapacitors. Nano Lett, 2010, 10: 42054031

9 Izadi-Najafabadi A, Yasuda S, Kobashi K, et al. Extracting the full potential of single-walled carbon nanotubes as durable supercapacitor electrodes operable at $4 \mathrm{~V}$ with high power and energy density. Adv Mater, 2010, 22: E235-E241

10 Serp P, Castillejos E. Catalysis in carbon nanotubes. ChemCatChem, 2010, 2: 41-47

11 Chen W, Fan Z L, Gu L, et al. Enhanced capacitance of manganese oxide via confinement inside carbon nanotubes. Chem Commun, 2010, 46: 3905-3907

12 Pan X L, Fan Z L, Chen W, et al. Enhanced ethanol production inside carbon-nanotube reactors containing catalytic particles. Nat Mater, 2007, 6: 507-511
13 Zhang J, Muller J O, Zheng W Q, et al. Individual Fe-Co alloy nanoparticles on carbon nanotubes: Structural and catalytic properties. Nano Lett, 2008, 8: 2738-2743

14 Guo S J, Pan X L, Gao H L, et al. Probing the electronic effect of carbon nanotube in catalysis: $\mathrm{NH}_{3}$ synthesis over $\mathrm{Ru}$ nanoparticles. Chem Eur J, 2010, 16: 5379-5384

15 Abbaslou R M M, Soltan J, Dalai A K. Effects of nanotubes pore size on the catalytic performances of iron catalysts supported on carbon nanotubes for Fischer-Tropsch synthesis. Appl Catal A-Gen, 2010, 379: $129-134$

16 Tessonnier J P, Ersen O, Weinberg G, et al. Selective deposition of metal nanoparticles inside or outside multiwalled carbon nanotubes. ACS Nano, 2009, 3: 2081-2089

17 Smirnov S, Vlassiouk I, Takmakov P, et al. Water confinement in hydrophobic nanopores. Pressure-induced wetting and drying. ACS Nano, 2010, 9: 5069-5075

18 Whitby M, Quirke N. Fluid flow in carbon nanotubes and nanopipes. Nat Nanotechnol, 2007, 2: 87-94

19 Kobayashi K, Suenaga K, Saito T, et al. Photoreactivity preservation of $\mathrm{AgBr}$ nanowires in confined nanospaces. Adv Mater, 2010, 22: 3156-3160

20 Cheng H M, Li F, Sun X, et al. Bulk morphology and diameter distribution of single-walled carbon nanotubes synthesized by catalytic decomposition of hydrocarbons. Chem Phys Lett, 1998, 289: 602610

21 Fischer J E, Dai H, Thess A, et al. Metallic resistivity in crystalline ropes of single-wall carbon nanotubes. Phys Rev B, 1997, 55: R4921R4924

22 Liu C, Cheng H M, Cong H T, et al. Synthesis of macroscopically long ropes of well-aligned single-walled carbon nanotubes. Adv Mater, 2000, 12: 1190-1192

23 Castillejos E, Debouttiere P J, Roiban L, et al. An efficient strategy to drive nanoparticles into carbon nanotubes and the remarkable effect of confinement on their catalytic performance. Angew Chem Int Ed, 2009, 48: 2529-2533

24 Wang C F, Guo S J, Pan X L, et al. Tailored cutting of carbon nanotubes and controlled dispersion of metal nanoparticles inside their Channels. J Mater Chem, 2008, 18: 5782-5786

25 Chen Z J, Guan Z H, Li M, et al. Enhancement of the performance of a platinum nanocatalyst confined within carbon nanotubes for asymmetric hydrogenation. Angew Chem Int Ed, 2011, 50: 1-6

26 Rinaldi A, Zhang J, Frank B, et al. Oxidative purification of carbon nanotubes and its impact on catalytic performance in oxidative dehydrogenation reactions. ChemSusChem, 2010, 3: 254-260

27 Zheng W Q, Zhang J, Zhu B, et al. Structure-function correlations for $\mathrm{Ru} / \mathrm{CNT}$ in the catalytic decomposition of ammonia. ChemSusChem, 2010, 3: 226-230

28 Garcia-Garcia F R, Alvarez-Rodriguez J, Rodriguez-Ramos I, et al. The use of carbon nanotubes with and without nitrogen doping as support for ruthenium catalysts in the ammonia decomposition reaction. Carbon, 2010, 48: 267-276

29 Kyotani T. Synthesis of various types of nano carbons using the template technique. Bull Chem Soc Jpn, 2006, 79: 2-1337

30 Masuda H, Hasegwa F, Ono S. Self-ordering of cell arrangement of anodic porous alumina formed in sulfuric acid solution. J Electrochem Soc, 197, 144: L127-L130

31 Martin C R. Membrane-based synthesis of nanomaterials. Chem Mater, 1996, 8: 1739-1746

32 Kyotani T, Tsai L F, Tomita A. Formation of ultrafine carbon tubes by using an anodic aluminum-oxide film as a template. Chem Mater 1995, 7: 1427-1428

33 Kyotani T, Tsai L F, Tomita A. Preparation of ultrafine carbon tubes in nanochannels of an anodic aluminum oxide film. Chem Mater 1996, 8: 2109-2113

34 Chen Z, Zhang H G. Mechanisms for formation of a one-dimensional horizontal anodic aluminum oxide nanopore array on a Si substrate. J Electrochem Soc, 2005, 152: D227 -D231

35 Thompson G E. Porous anodic alumina: Fabrication, characterization and applications. Thin Solid Films, 1997, 297: 192-201 
36 Li F Y, Zhang L, Metzger R M. On the growth of highly ordered pores in anodized aluminum oxide. Chem Mater, 1998, 10: 24702480

37 Yuan Z H, Huang H, Liu L, et al. Controlled growth of carbon nanotubes in diameter and shape using template-synthesis method. Chem Phys Lett, 2001, 345: 39-43

38 Ciambelli P, Arurault L, Sarno M, et al. Controlled growth of CNT in mesoporous AAO through optimized conditions for membrane preparation and CVD operation. Nanotechnology, 2011, 22: 265613

39 Hillebrenner H, Buyukserin F, Stewart J D, et al. Template synthesized nanotubes for biomedical wangwang applications. Nanomedicine, 2006, 1: 39-50

40 Ittisanronnachai S, Orikasa H, Inokuma N, et al. Small molecule delivery using carbon nano-test-tubes. Carbon, 2008, 46: 1361-1363

41 Wang X H, Orikasa H, Inokuma N, et al. Controlled filling of Permalloy into one-end-opened carbon nanotubes. J Mater Chem, 2007, 17: 986-991

42 Che G L, Lakshmi B B, Fisher E R, et al. Carbon nanotubule membranes for electrochemical energy storage and production. Nature, 1998, 393: 346-349

43 Mao Y B, Park T J, Zhang F, et al. Environmentally friendly methodologies of nanostructure synthesis. Small, 2007, 3: 1122-1139

44 Keller F, Hunter M S, Robinson D L. Structural features of oxide coatings on aluminium. J Electrochem Soc, 1953, 100: 411-419

45 Bocchetta G D, Parkola K G. Temperature influence on well-ordered nanopore structures grown by anodization of aluminium in sulphuric acid. Electrochim Acta, 2007, 52: 1880-1888

46 Osullivan J P, Wood G C. Morphology and mechanism of formation pf porous anodic films on aluminium. Proc R Soc Lond Ser A, 1970, 317: 511-543

47 Paolini G, Masoero M, Sacchi F, et al. An investigation of porous anodic oxide films on aluminum by comparative adsorption gravimetric and electronoptical measurements. J Electrochem Soc, 1965, 112: 32-38

48 Parkhutik V P, Shershulsky V I. Theoretical modeling of porous oxidegrowth on aluminum. J Phys D-Appl Phys, 1992, 25: 1258-1263

49 Furneaux R C, Rigby W R, Davidson A P. The formation of controlled-porosity membranes from anodically oxidized aluminum. Nature, 1989, 337: 147-149

50 Bocchetta P, Sunseri C, Bottino A, et al. Asymmetric alumina membranes electrochemically formed in oxalic acid solution. J Appl Electrochem, 2002, 32: 977-985

51 Palibroda E. Aluminum porous oxide growth II. On the rate determining step. Electrochim Acta, 1995, 40: 1051-1055

52 Wood G C, O'Sullivan J P. The anodizing of aluminium in sulphate solutions. Electrochim Acta, 1970, 15: 1865-1876

53 Yu M, Funke H H, Falconer J L, et al. Noble, high density, verticallyaligned carbon nanotube membranes, Nano Lett, 2009, 9: 225-229

54 Srivastava A, Srivastava O N, Talapatra S, et al. Carbon nanotube filters. Nat Mater, 2004, 3: 610-614

55 Schneider J J, Engstler N, Budna K P, et al. Freestanding, highly flexible, large area, nanoporous alumina membranes with complete through-hole pore morphology. Eur J Inorg Chem, 2005, 12: 23522359

56 Mata-Zamora M E, Saniger J M. Thermal evolution of porous anodic alumina: A comparative study. Rev Mex Fis, 2005, 51: 502-509

57 Sulka G D, Stroobants S, Moshchalkov V, et al. Synthesis of wellordered nanopores by anodizing aluminum foils in sulfuric acid. J Electrochem Soc, 2002, 149: D97-D103

$58 \mathrm{Xu} \mathrm{W} \mathrm{L,} \mathrm{Zheng} \mathrm{M} \mathrm{J,} \mathrm{Wu} \mathrm{S,} \mathrm{et} \mathrm{al.} \mathrm{Effects} \mathrm{of} \mathrm{high-temperature}$ annealing on structural and optical properties of highly ordered porous alumina membranes. Appl Phys Lett, 2004, 85: 4364-4366

59 Nakamura S, Saito M, Huang L F, et al. Infrared optical-constants of anodic alumina films with micropore arrays. Jpn J Appl Phys, 1992, 31: 3589-3593

60 Rehn L E, Kestel B J, Baldo P M, et al. Self-organized porousalumina implantation masks for generating nanoscale arrays. Nucl Instrum Methods Phys Res Sect B-Beam Interact Mater Atoms, 2003, 206: 490-494
61 Li A P, Muller F, Birner A, et al. Hexagonal pore arrays with a 50-420 $\mathrm{nm}$ interpore distance formed by self-organization in anodic alumina. J Appl Phys, 1998, 84: 6023-6026.

62 Yan J C, Rao G V R, Barela M, et al. Growth of patterned nanopore arrays of anodic aluminum oxide. Adv Mater, 2003, 15: 2015-2018

$63 \mathrm{Xu}$ T, Zangari G, Metzger R M. Periodic holes with $10 \mathrm{~nm}$ diameter produced by grazing $\mathrm{Ar}^{+}$milling of the barrier layer in hexagonally ordered nanoprous alumina. Nano Lett, 2002, 2: 37-41

64 Yuan J H, Chen W, Hui R J, et al. Mechanism of one-step voltage pulse detachment of porous anodic alumina membranes. Electrochim Acta, 2006, 51: 4589-4595

65 Yuan J H, He F Y, Sun D C, et al. A simple method for preparation of through-hole porous anodic alumina membrane. Chem Mater, 2004, 16: 1841-1844

66 Parthasarathy R V, Phani K L N, Martin C R. Template synthesis of graphitic nanotubules. Adv Mater, 1995, 7: 896-897

67 Chen J T, Shin K, Leiston-Belanger J M, et al. Amorphous carbon nanotubes with tunable properties via template wetting. Adv Funct Mater, 2006, 16: 1476-1480

68 Schneider J J, Engstler J. Carbon and polymer filaments in nanoporous alumina. Eur J Inorg Chem, 2006, 9: 1723-1736

69 Schneider J J, Maksimova N I, Engstler J, et al. Catalyst free growth of a carbon nanotube-alumina composite structure. Inorg Chim Acta, 2008, 361: 1770-1778

70 Wang X H, Akahane T, Orikasa $\mathrm{H}$, et al. Brilliant and tunable color of carbon-coated thin anodic aluminum oxide films. Appl Phys Lett, 2007, 91: 011908

71 Che G, Lakshmi B B, Martin C R, et al. Chemical vapor deposition based synthesis of carbon nanotubes and nanofibers using a template method. Chem Mater, 1998, 10: 260-267

72 Ciambelli P, Sannino D, Sarno M, et al. Hydrocarbon decomposition in alumina membrane: An effective way to produce carbon nanotubes bundles. J Nanosci Nanotechnol, 2004, 4: 779-787

73 Ciambelli P, Sannino D, Sarno M, et al. Characterization of nanocarbons produced by CVD of ethylene in alumina or aluminosilicate matrices. Adv Eng Mater, 2004, 6: 804-811

74 Nagayama M, Tamura K. Dissolution of anodic oxide film on aluminium in a sulphuric acid solution. Electrochim Acta, 1967, 12: 1097-1107

75 Nagayama M, Tamura K, Takahashi H. Mechanism of open-circuit dissolution of porous oxide films on aluminium in acid solutions. Corros Sci, 1972, 12: 133-136

76 Orikasa H, Inokuma N, Okubo S, et al. Template synthesis of waterdispersible carbon nano "test tubes" without any post-treatment. Chem Mater, 2006, 18: 1036-1040

77 Wade T L, Wegrowe J E. Template synthesis of nanomaterials. Eur Phys J Appl Phys, 2005, 29: 3-22

78 Chik H, Xu J M. Nanometric superlattices: Non-lithographic fabrication, materials, and prospects. Mater Sci Eng R, 2004, 43: 103-138

79 Miao Y, Cai Y, Chan Y F, et al. A novel carbon nanotube structure formed in ultra-long nanochannels of anodic aluminum oxide templates. J Phys Chem B, 2006, 110: 2080-2083

80 Sigurdsona S, Sundaramurthya V, Dalaia A K, et al. Effect of anodic alumina pore diameter variation on template-initiated synthesis of carbon nanotube catalyst supports. J Mol Catal A: Chem, 2009, 306: 23-32

81 Zhao N H, Zhang P, Yang L C, et al. Tunable amorphous carbon nanotubes prepared by a simple template. Mater Lett, 2009, 63: 1955-1957

82 Tian M L, Xu S Y, Wang J G, et al. Penetrating the oxide barrier in situ and separating freestanding porous anodic alumina films in one step. Nano Lett, 2005, 5: 697-703

83 Iijima S. Helical microtubules of graphitic carbon. Nature, 1991, 354: 56-58

84 Cheng H M, Li F, Su G, et al. Large-scale and low-cost synthesis of single-walled carbon nanotubes by the catalytic pyrolysis of hydrocarbons. Appl Phys Lett, 1998, 72: 3282-3284

85 Thess A, Lee R, Nikolaev P, et al. Crystalline ropes of metallic carbon nanotubes. Science, 1996, 273: 483-487 
86 Diggle J W, Downie T C, Goulding C W. Anodic oxide films on Aluminum. Chem Rev, 1969, 69: 365-405

87 Papadopoulos C, Rakitin A, Li J, et al. Electronic transport in Y-junction carbon nanotubes. Phys Rev Lett, 2000, 85: 3476-3479

88 Meng G W, Jung Y J, Cao A Y, et al. Controlled fabrication of hierarchically branched nanopores, nanotubes, and nanowires. Proc Natl Acad Sci USA, 2005, 102: 7074-7078

89 Meng G W, Han F M, Zhao X L, et al. A general synthetic approach to interconnected nanowire/nanotube and nanotube/nanowire/nanotube heterojunctions with branched topology. Angew Chem Int Ed, 2009, 48: 7166-7170

90 Li J, Papadopoulos C, Xu J. Nanoelectronics: Growing Y-junction carbon nanotubes. Nature, 1999, 402: 253-254

91 Sui Y C, Gonzalez-Leon J A, Bermudez A, et al. Synthesis of multi branched carbon nanotubes in porous anodic aluminum oxide template. Carbon, 2001, 39: 1709-1715

92 Saito S. Carbon nanotubes for next-generation electronic devices. Science, 1997, 278: 77-78

93 Yang Q H, Xu W H, Tomita A, et al. Double coaxial structure and dual physicochemical properties of carbon nanotubes composed of stacked nitrogen-doped and undoped multiwalls. Chem Mater, 2005, 17: 2940-2945

94 He M S, Zhou S, Zhang J, et al. CVD growth of $n$-doped carbon nanotubes on silicon substrates and its mechanism. J Phys Chem B, 2005, 109: 9275-9279

95 Liu J W, Webster S, Carroll D L. Temperature and flow rate of $\mathrm{NH}_{3}$ effects on nitrogen content and doping environments of carbon nanotubes grown by injection CVD method. J Phys Chem B, 2005, 109: $15769-15774$

96 Lee Y T, Kim N S, Bae S Y, et al. Growth of vertically aligned nitrogen-doped carbon nanotubes: Control of the nitrogen content over the temperature range $900-1100^{\circ} \mathrm{C}$. J Phys Chem B, 2003, 107: 12958-12963

97 Brown B, Parker C B, Stoner B R, et al. Growth of vertically aligned bamboo-like carbon nanotubes from ammonia/methane precursors using a platinum catalyst. Carbon, 2011, 49: 266-274

98 Yang Q H, Hou P X, Unno M, et al. Dual Raman features of double coaxial carbon nanotubes with $\mathrm{N}$-doped and B-doped multiwalls. Nano Lett, 2005, 12: 2465-2469

99 Kwon T, Nishihara H, Itoi $\mathrm{H}$, et al. Enhancement mechanism of electrochemical capacitance in nitrogen- /boron-doped carbons with uniform straight nanochannels. Langmuir, 2009, 25: 11961-11968

100 Yang Q H, Xu W H, Tomita A, et al. The template synthesis of double coaxial carbon nanotubes with nitrogen-doped and boron-doped multiwalls. J Am Chem Soc, 2005, 127: 8956-8957

101 Xu W H, Kyotani T, Pradhan B K, et al. Synthesis of aligned carbon nanotubes with double coaxial structure of nitrogen-doped and undoped multiwalls. Adv Mater, 2003, 15: 1087-1090

102 Yu S S, Zheng W T. Effect of N/B doping on the electronic and field emission properties for carbon nanotubes, carbon nanocones, and graphene nanoribbons. Nanoscale, 2010, 2: 1069-1082

103 Xu Z, Lu W G, Wang W L, et al. Converting metallic single-walled carbon nanotubes into semiconductors by boron/nitrogen co-doping. Adv Mater, 2008, 20: 3615-3619

104 Yu D S, Zhang Q, Dai L M. Highly efficient metal-free growth of nitrogen-doped single-walled carbon nanotubes on plasma-etched substrates for oxygen reduction. J Am Chem Soc, 2010, 132: 1512715129

105 Ayala P, Plank W, Gruneis A, et al. A one step approach to B-doped single-walled carbon nanotubes. J Mater Chem, 2008, 18: 5676-5681

106 Czerw R, Terrones M, Charlier J C, et al. Identification of electron donor states in N-doped carbon nanotubes. Nano Lett, 2009, 9: 457460

107 Cruz-Silva E, Cullen D A, Gu L, et al. Heterodoped nanotubes: Theory, synthesis, and characterization of phosphorus-nitrogen doped multiwalled carbon nanotubes. ACS Nano, 2008, 2: 441-448

108 Glerup M, Castignolles M, Holzinger M, et al. Synthesis of highly nitrogen-doped multi-walled carbon nanotubes. Chem Commun, 2003, 39: 2542-2543
109 Zhao X W, Jiang P, Xie S S. Aligned Ni nanoparticle arrays encapsulated in carbon nanotubes. Solid State Commun, 2009, 149: 1984-1987

$110 \mathrm{Hu}$ J, Bando Y, Zhan J, et al. Carbon nanotubes as nanoreactors for fabrication of single-crystalline $\mathrm{Mg}_{3} \mathrm{~N}_{2}$ nanowires. Nano Lett, 2006, 6: 1136-1140

111 Ugarte D, Chatelain A, deHeer W A. Nanocapillarity and chemistry in carbon nanotubes. Science, 1996, 274: 1897-1899

112 Ajayan P M, Iijima S. Capillarity-induced filling of carbon nanotubes. Nature, 1993, 361: 333-334

113 Tsang S C, Chen Y K, Harris P J, et al. A simple chemical method of opening and filling carbon nanotubes. Nature, 1994, 372: 159162

114 Ebbesen T W. Wetting, filling and decorating carbon nanotubes. J Phys Chem Solids, 1996, 57: 951-955

115 Ma H X, Wang L C, Chen L Y, et al. Pt nanoparticles deposited over carbon nanotubes for selective hydrogenation of cinnamaldehyde. Catal Commun, 2007, 8: 452-456

116 Winter F, Bezemer G L, vand der Spek C, et al. TEM and XPS studies to reveal the presence of cobalt and palladium particles in the inner core of carbon nanofibers. Carbon, 2005, 43: 327-332

117 Costa P M F J, Friedrichs S, Sloan J, et al. Imaging lattice defects and distortions in alkali-metal iodides encapsulated within doublewalled carbon nanotubes. Chem Mater, 2005, 17: 3122-3129

118 Yu W J, Hou P X, Zhang L L, et al. Preparation and electrochemical property of $\mathrm{Fe}_{2} \mathrm{O}_{3}$ nanoparticles-filled carbon nanotubes. Chem Commun, 2010, 46: 8576-8578

119 Pelletier V, Bhattacharyya S, Knoke I, et al. Copper azide confined inside templated carbon nanotubes. Adv Funct Mater, 2010, 20: 3168-3174

120 Kyotani T, Pradhan B K, Tomita A. Synthesis of carbon nanotube composites in nanochannels of an anodic aluminum oxide film. Bull Chem Soc Jpn, 1999, 72: 1957-1970

121 Kyotani T, Tsai L F, Tomita A. Formation of platinum nanorods and nanoparticles in uniform carbon nanotubes prepared by a template carbonization method. Chem Commun, 1997, 33: 701-702

122 Costa P M F J, Sloan J, Rutherford T, et al. Encapsulation of $\operatorname{Re}_{x} \mathrm{O}_{y}$ clusters within single-walled carbon nanotubes and their in tubulo reduction and sintering to Re metal. Chem Mater, 2005, 17: 65796582

123 Schulte K, Swarbrick J C, Smith N A, et al. Assembly of cobalt phthalocyanine stacks inside carbon nanotubes. Adv Mater, 2007, 19: 3312-3316

124 Schmid G. Materials in nanoporous alumina. J Mater Chem, 2002, 12: $1231-1238$

125 Pradhan B K, Toba T, Kyotani T, et al. Inclusion of crystalline iron oxide nanoparticles in uniform carbon nanotubes prepared by a template carbonization method. Chem Mater, 1998, 10: 2510-2515

126 Matsui K, Pradhan B K, Kyotani T, et al. Formation of nickel oxide nanoribbons in the cavity of carbon nanotubes. J Phys Chem B, 2001, 105: 5682-5688

127 Pradhan B K, Kyotani T, Tomita A. Nickel nanowires of $4 \mathrm{~nm}$ diameter in the cavity of carbon nanotubes. Chem Commun, 1999, 35: 1317-1318

128 Orikasa H, Inokuma N, Ittisanronnachai S, et al. Template synthesis of water-dispersible and magnetically responsive carbon nano test tubes. Chem Commun, 2008, 44: 2215-2217

129 Kim K H, Yamaguchi M, Orikasa H, et al. Broadband GHz frequency characterizations of Permalloy nanorods' array. Solid State Commun, 2006, 140: 491-494

130 Liu L F, Mu S C, Xie S S, et al. Template synthesis, characterization and magnetic property of $\mathrm{Fe}$ nanowires-filled amorphous carbon nanotubes array. J Phys D-Appl Phys, 2006, 39: 3939-3944

131 Bao J C, Zhou Q F, Hong J M, et al. Synthesis and magnetic behavior of an array of nickel-filled carbon Nanotubes. App Phys Lett, 2002, 81: 4592-4594

132 Gautam U K, Costa P M F J, Bando Y, et al. Recent developments in inorganically filled carbon nanotubes: Successes and challenges. Sci Technol Adv Mater, 2010, 11: 054501 
133 Tang D M, Yin L C, Li F, et al. Carbon nanotube-clamped metal atomic chain. Proc Natl Acad Sci USA, 2010, 107: 9055-9059

134 Sui Y C, Acosta D R, Gonzalez-Leon J A, et al. Structure, thermal stability, and deformation of multibranched carbon nanotubes synthesized by CVD in the AAO template. J Phys Chem B, 2001, 105: 1523-1527

135 Hornyak G L, Dillon A C, Parilla P A, et al. Template synthesis of carbon nanotubes. Nanostruct Mater, 1999, 12: 83-88

136 Babaa M R, McRae E, Delpeux S, et al. Surface characterization of template-synthesized multi-walled carbon nanotubes. Chem Phys Lett, 2004, 396: 49-53

137 Delpeux-Ouldriane S, Szostak K, Frackowiak E, et al. Annealing of template nanotubes to well-graphitized multi-walled carbon nanotubes. Carbon, 2006, 44: 799-823

138 Chen P L, Chang J K, Pan F M, et al. Tube number density control of carbon nanotubes on anodic aluminum oxide template. Diamond Relat Mater, 2005, 14: 804-809

139 Choi W B, Cheong B H, Kim J H, et al. Selective growth of carbon nanotubes for nanoscale transistors. Adv Funct Mater, 2003, 13: 8084

140 Xu J M, Zhang X B, Chen F, et al. Preparation and modification of well-aligned CNTs, grown on AAO template. Appl Surf Sci, 2005, 239: $320-326$

141 Jeong S H, Lee K. Fabrication of the aligned and patterned carbon nanotube field emitters using the anodic aluminum oxide nano-template on a Si wafer. Synth Met, 2003, 139: 385-390

142 Vicente L, Carmen M, Francisco M, et al. Anodic aluminium oxide membranes used for the growth of carbon nanotubes. J Nanosci Nanotechnol, 2009, 9: 6396-6400

143 Chen W, Fan Z L, Pan X L, et al. Effect of confinement in carbon nanotubes on the activity of Fischer-Tropsch iron catalyst. J Am Chem Soc, 2008, 130: 9414-9419

144 Hulteen J C, Martin C R. A general template-based method for the preparation of nanomaterials. J Mater Chem, 1997, 7: 1075-1087

145 Inada T, Uno N, Kato T, et al. Meso-porous alumina capillary tube as a support for high-temperature gas separation membranes by novel pulse sequential anodic oxidation technique. J Mater Res, 2005, 20: $114-120$

146 Wang S R, Liang Z Y, Wang B, et al. Precise cutting of singlewalled carbon nanotubes. Nanotechnology, 2007, 18: 055301-055306

147 Ziegler K J, Gu Z N, Shaver J, et al. Cutting single-walled carbon nanotubes. Nanotechnology, 2005, 16: S539-S544

Open Access This article is distributed under the terms of the Creative Commons Attribution License which permits any use, distribution, and reproduction in any medium, provided the original author(s) and source are credited. 